\title{
Ein Recht auf Hoffnung aus Art. 3 EMRK: Lebenslange Freiheitsstrafen in Europa
}

\author{
Christine Morgenstern, Greifswald"
}
A. Einleitung
153
B. Recht und Praxis lebenslanger Frei- heitsstrafen und sehr langer zeitiger Freiheitsstrafen ..................... 155
I. Strafrahmen und Strafzumessung . . 155
II. Strafzwecke bei der lebenslangen und den sehr langen zeitigen Freiheits- strafen
III. Strafrestaussetzung bei lebenslangen Freiheitsstrafen und ihre Begrün- dung
C. Europäisches Recht: Die EMRK und die Rechtsprechung des EGMR ....... 173
I. Die Grundlagen der EMRK ......... 173

II. Die Entwicklung der EGMR-Rechtsprechung zu nicht aussetzbaren lebenslangen Freiheitsstrafen....... 176

III. Die Entscheidung der Großen Kammer des EGMR im Fall Vinter u.a.. . 177

D. Würdigung: Das Recht auf Hoffnung als europäisches Menschenrecht? ...... 179 I. Die Herleitung aus Art. 3 EMRK .... 179

II. Entlassungsaussicht de jure und de facto: spezialpräventive Kriterien. . . 181

III. Die prozessuale Absicherung der Aussicht auf Entlassung............ 182

IV. Konsequenzen .................... 185

E. Ausblick ......................... 187

\section{A. Einleitung}

Ein Mann hat in 54 Fällen Menschen zu Tötung in den Gaskammern von Auschwitz ausgesucht. Ein Mann hat wegen der Aussicht auf eine reiche Erbschaft seine Adoptiveltern, seine Schwester und deren sechsjährige Zwillinge erschossen. Ein Mann hat sein Opfer, einen umstrittenen Künstler, auf offener Straße angeschossen und ihm anschließend die Kehle durchgeschnitten. Eine Frau war an einem Bombenanschlag beteiligt, bei dem 14 Menschen starben und 78 verletzt wurden. Ein Mann hat insgesamt 138 Menschen teils erschossen, teils in verbarrikadierten Häusern verbrennen lassen. Diese Beispiele schwerster Verbrechen aus Deutschland, England, den Niederlanden, Spanien und Bosnien wurden mit Höchststrafen geahndet - in Deutschland, England und den Niederlanden mit lebenslangen Freiheitsstrafen, in Spanien mit einer aus den Einzeltaten errechneten Freiheitsstrafe von insgesamt 2232 Jahren. Im letzten skizzierten Fall wurde der Täter vom Internationalen Strafgerichtshof für das ehemalige Jugoslawien zu lebenslanger Freiheitsstrafe verurteilt. ${ }^{1}$

Der vorliegende Beitrag beschäftigt sich mit der Frage, ob und mit welcher Begründung auch in Fällen wie den beschriebenen eine bedingte Entlassung aus der Haft in Frage kommt. Dabei sind Strafzweckerwägungen, kriminalpolitische Entwicklungen

* Dr. Christine Morgenstern ist wissenschaftliche Mitarbeiterin am Lehrstuhl für Kriminologie der ErnstMoritz-Arndt-Universität Greifswald.

1 Die Fälle sind verschiedenen Urteilen entlehnt: BVerfGE 72, 105 (NJW 1986, 2241); EGMR (Große Kammer, GK) vom 9.7.2013, Vinter u.a./Vereinigtes Königreich, No. 66069/09,130/10 and 3896, unter dem zweiten Aktenzeichen des Beschwerdeführers Bamber; Distriktgericht Amsterdam, Urteil vom 26. Juli 2005, zu finden als LJN AU0025 580 in der niederländischen Rechtsprechungsdatenbank www.rechtspraak.nl (Fall Theo van Gogh); EGMR (GK) vom 21.10.2013, Del Rio Prada./. Spain, No. 42750/09; sowie International Criminal Tribunal for the former Yugoslavia (ICTY) Prosecutor v. Milan Lukić and Sredoje Lukić, Case No. IT-98-32/1-A, Appeal Chamber, 4 December 2012. 
sowie materielle Geltung und prozessuale Absicherung von Grund- und Menschenrechten zu diskutieren. Aktueller Hintergrund der Fragestellung ist das Urteil des Europäischen Gerichtshofs für Menschenrechte (EGMR) im Fall Vinter u.a., ${ }^{2}$ in dem ein Verstoß gegen Art. 3 EMRK (das Verbot der Folter, unmenschlicher oder erniedrigender Behandlung oder Strafe) durch die englische Praxis der sog. whole life order angenommen wurde. Diese Variante der lebenslangen Freiheitsstrafe ist nicht aussetzbar. Zentral war dabei das Argument, dass die Betroffenen in einem dauerhaft hoffnungslosen Zustand leben, wenn die Möglichkeit einer Entlassung und die Möglichkeit einer entsprechenden Haftprüfung nicht bestehen. In Deutschland gilt dieses Problem seit 1977 mit dem „Lebenslang-Urteil“ des Bundesverfassungsgerichts als geklärt: ${ }^{3}$ Auch die lebenslange Freiheitsstrafe muss prinzipiell aussetzbar sein, die Wiedererlangung der Freiheit kann nur dann auf Dauer verwehrt werden, wenn andernfalls die Gefahr von Wiederholungstaten besteht. In einer Minderheit europäischer Staaten, neben England/Wales ${ }^{4}$ auch in den Niederlanden, gibt es eine solche Möglichkeit der vorzeitigen Beendigung jedoch nicht. In einer weiteren Minderheit europäischer Staaten, darunter Spanien, gibt es keine lebenslange Freiheitsstrafe, dafür aber die Möglichkeit, sehr lange zeitige Freiheitsstrafen zu vollstrecken. Eine spezifische Praxis hat zudem der Internationalen Strafgerichtshof für das ehemalige Jugoslawien entwickelt, der sich zuständigkeitshalber ausschließlich mit schwersten Verbrechen beschäftigt.

Mitunter wird dennoch ein „spezifisch europäisches Humanitätsverständnis“ und eine „europäische Strafrechtsidentität“ angenommen, die sich gerade durch die „Ächtung extremer Strafen wie der Todesstrafe oder der bis zum Tod ohne Begnadigungsaussicht vollzogen Freiheitsstrafe " auszeichnen. ${ }^{5}$ Das Vinter - Urteil gibt daher sowohl Anlass zur Frage nach der Konturierung des Art. 3 EMRK als auch nach der Existenz eines solchen europäischen Konsenses in Bezug auf die lebenslange Freiheitsstrafe. Der vorliegende Beitrag beleuchtet dafür zunächst die strafrechtliche Seite, d. h. die rechtlichen Voraussetzungen für die Verhängung lebenslanger oder sehr langer Freiheitsstrafen und die Modalitäten ihrer Aussetzung oder anderer Beendigungsgründe. Ergänzend werden jeweils einige Daten zur quantitativen Bedeutung in der jeweiligen nationalen Praxis und der des Jugoslawien-Strafgerichtshofs

2 EGMR, Vinter u.a../. Großbritannien (GK), (Fn. 1).

3 BVErfGE 45, 187; G. Kett-Straub 2011, Die lebenslange Freiheitsstrafe: Legitimation, Praxis, Strafrestaussetzung und besondere Schwere der Schuld, Tübingen 2011, S. 46 ff. und $93 \mathrm{ff}$.

4 Großbritannien als Union von England, Wales, Schottland und Nordirland hat drei verschiedene Justizssysteme, England und Wales bilden hier eine Einheit. Im vorliegenden Beitrag geht es ebenso wie im Fall Vinter u.a. nur um englisch/walisisches Recht.

5 J. Vogel, Europäische Kriminalpolitik - europäische Strafrechtsdogmatik, GA 2002, S. 519 (522). Ähnlich, aber skeptischer, D. van Zyl Smit, Outlawing Irreducible Life Sentences: Europe on the Brink? Federal Sentencing Reporter 23, S. 39 (39 f.) und S. Snacken/D. Van Zyl Smit, Distinctive Features of European Penology and Penal Policy-Making, in: T. Daems/D. van Zyl Smit/S. Snacken (Hrsg.), European Penology? Oxford/Portland 2013, S. $10 \mathrm{ff}$. 
vorgestellt. $^{6}$ Anschließend wird die Entwicklung der Rechtsprechung zu Art. 3 EMRK in dieser Fragestellung nachvollzogen, bevor vor dem ausgebreiteten Hintergrund das Vinter-Urteil gewürdigt und in einem Ausblick seine Konsequenzen aufgezeigt werden.

\section{B. Recht und Praxis lebenslanger Freiheitsstrafen und sehr langer zeitiger Freiheitsstrafen}

\section{Strafrahmen und Strafzumessung}

Die skizzierten Fälle sind als Morde abgeurteilt worden, teilweise kamen terroristische Delikte und Delikte aus dem Völkerstrafrecht hinzu. Mord wird in Deutschland gem. \211 StGB zwingend mit eine lebenslangen Freiheitsstrafe bestraft. Durch das Tatgericht kann außerdem die besondere Schwere der Schuld festgestellt werden ( $\int 57 \mathrm{a}$ StGB), um dem Umstand Rechnung zu tragen, dass auch bei den schwersten Straftaten noch verschiedene Schweregrade vorkommen - bei Mord etwa, wenn mehrere Opfer zu beklagen sind oder die Tat unter besonders abscheulichen Begleitumständen ausgeführt wird. Wegen des Mehr bei den Tatumständen, aus denen sich das Mehr an Schuld ergibt, wird die Vorschrift auch als „Quasi-Qualifikation“ aufgefasst. ${ }^{7}$ Wird die besondere Schwere der Schuld festgestellt, entfällt nicht die Möglichkeit der bedingten Entlassung, in aller Regel erfolgt sie aber deutlich später als nach der ansonsten geltenden Mindestverbüßungsdauer von 15 Jahren.

Jährlich werden in Deutschland rund 100 Menschen zu lebenslangen Freiheitsstrafen verurteilt, ${ }^{8}$ das sind ca. $0,08 \%$ aller strafrechtlichen Verurteilungen. ${ }^{9}$ Fast alle Fälle betreffen Morde oder Mordversuche. Trotz der relativen Stabilität dieses Ausgangswertes hat sich die Zahl derjenigen Gefängnisinsassen, die ihre Strafe als „Lebenslängliche“ verbüßen, stetig erhöht: 2012 waren es 2031, im Jahr 1992, dem ersten Jahr der Datenerhebung für das ganze Bundesgebiet, waren es noch 1294 gewe-

6 Dabei wird die Unterbringung von Personen, die allein wegen fortbestehender Gefährlichkeit inhaftiert sind, außer Betracht bleiben, weil dieses Thema von anderen rechtlichen und tatsächlichen Besonderheiten betroffen ist. In Deutschland betrifft dies die Sicherungsverwahrung. In England sind dies diejenigen, bei denen eine die unbestimmte Freiheitsstrafe zum Schutz der Öffentlichkeit, „Imprisonment for Public Protection (IPP)“, angeordnet ist. Sie sind derzeit noch sehr zahlreich, seit 2012 allerdings wird diese Sanktion nicht mehr verhängt. In den Niederlanden gibt es die Maßregel der Überlassung zur staatlichen Behandlung (,Terbeschikkingsstelling“(Tbs), $\mathbb{3} 7$ lit. b Wetboek van Strafrecht), die, grob umschrieben, eine Mischform aus deutscher Maßregel nach $\$ 63$ StGB und Sicherungsverwahrung darstellt und die wegen ihrer Verlängerungsmöglichkeiten ebenfalls als unbestimmte Freiheitsentziehung anzusehen ist.

7 K. Lüderssen, Anmerkung zur Entscheidung des Bundesgerichtshofs vom 2.2.2005, StV 2006, S. 61 (61).

8 Die Zahlen schwanken zwischen 2007 und 2011 zwischen 94 Verurteilungen (im Jahr 2011) und 137 (im Jahr 2010) im ganzen Bundesgebiet (Quelle: Statistisches Bundesamt, Strafverfolgungsstatistik). Zur Entwicklung in den 1990er Jahren C. Morgenstern, „Lebenslang“ ernst nehmen - Die Praxis der lebenslangen Freiheitsstrafe, Neue Kriminalpolitik 2004, S. 52 ff.

9 Nach allgemeinem Strafrecht, ohne Straßenverkehrsdelikte (vgl. zur Strafzumessungspraxis ausführlich F. Dünkel, F./C. Morgenstern, Deutschland. In: F. Dünkel/T. Lappi-Seppälä/C. Morgenstern/D. van Zyl Smit [Hrsg.], Kriminalität, Kriminalpolitik, strafrechtliche Sanktionspraxis und Gefangenenraten im europäischen Vergleich, Mönchengladbach 2010, S. 97[131 ff.]). 
sen. ${ }^{10}$ Für das Jahr 2012 lässt sich ein Anteil von 3\% an allen Gefangenen und von $3,5 \%$ an allen Strafgefangenen berechnen. Wie viele lebenslange Freiheitsstrafe anteilig ausgesetzt werden (und wann), lässt sich nicht mit Sicherheit beantworten. Eine Längsschnittbetrachtung derjenigen, bei denen die lebenslange Haft durch bedingte Entlassung oder auf anderen Weise (durch Tod, Begnadigung oder Ausweisung bzw. Überstellung) beendet wurde, ist jedoch möglich. ${ }^{11}$ Danach betrug der Median der Haftdauer der zwischen 2007 und 2009 Entlassenen rund 16 Jahre. Betrachtet man den letzten ausgewerteten Jahrgang 2010 isoliert, waren die meisten (45 von 90) Entlassenen zwischen 15 und 20 Jahren inhaftiert, weitere 21 länger als 20 Jahre, einer davon 45 Jahre.

In England und Wales muss Mord ebenfalls mit lebenslanger Freiheitsstrafe geahndet werden (sec. 8 Murder [Abolition of the Death Penalty] Act von 1965). Auch hier sind zwei verschiedene Varianten möglich. In der Regel wird eine Mindestverbüßungszeit, der sog. tariff, festgesetzt. Bei Mord geht das englische Recht hierfür bei Tätern über 21 Jahren von einer Regelverbüßungsdauer von 15 Jahren aus (Criminal Justice Act [CJA] 2003, sec. 269-277 und Strafzumessungsrichtlinie „Schedule 21: Determination of minimum term in relation to mandatory life sentences“). In Fällen, bei denen eine Waffe zum Einsatz kam, sind es 25 Jahre; bei schwerer wiegenden Begleitumständen sind es 30 Jahre. Wenn das Tatgericht jedoch der Auffassung ist, dass der Schweregrad außergewöhnlich hoch (exceptionally high) ist, ist eine nicht aussetzbare lebenslange Freiheitsstrafe möglich, die whole life order. Diese Kategorie beinhaltet z. B. mehrfachen Mord, wenn jeder Fall besonders kaltblütig geplant wurde, die Entführung der Opfer voranging oder die Morde einen sexuellen bzw. sadistischen Hintergrund hatten; Sexualmord an Kindern; Mord zur Verfolgung eines politischem, religiösem oder ideologischem Ziels oder ein Mord, begangen durch einen bereits wegen Mordes Verurteilten. Es gibt Bestrebungen, künftig auch Polizistenmord in diese Kategorie aufzunehmen. ${ }^{12}$

Jährlich werden deutlich mehr Menschen zu lebenslanger Haft verurteilt als in Deutschland - zuletzt waren es 426, in den zehn Jahren zuvor bewegten sich die Zahlen zwischen 400 und gut 600. ${ }^{13}$ Ende März 2013 verbüßten 7576 Menschen eine lebenslange Freiheitsstrafe, ihr Anteil an allen Gefangenen beträgt $9 \%$, an allen

10 Quelle: Statistisches Bundesamt, Strafvollzugstatistik, jeweils zum 31.3. eine Jahres und eigene Berechnungen.

11 Untersucht werden können auf die verbüßte Strafzeit hin nur die Entlassenen, vgl. K. Laubenthal, Lebenslange Freiheitsstrafe: Vollzug und Aussetzung des Strafrestes zur Bewährung, Lübeck 1987, S. 106. Seit 2002 wird eine detaillierte Auswertung von Daten durch die Kriminologische Zentralstelle durchgeführt, vgl. A. Dessecker, Lebenslange Freiheitsstrafe und Sicherungsverwahrung, Dauer und Gründe der Beendigung im Jahr 2010, Wiesbaden 2012, S. 8 f.

12 Pressemitteilung der britischen Regierung vom 15.5.2013: „Theresa May: murderers of police to receive whole-life jail sentences”, www.gov.uk.

13 Ministry of Justice, Statistics bulletin: Criminal Justice Statistics, Quarterly Update to December 2013 (www.gov.uk, abgerufen am 10.4.2014), S. 48. 
Strafgefangenen über $10 \% .{ }^{14}$ Nur 48 verbüßten jedoch eine whole life order, wobei dieser Stichtagswert ist in den letzten Jahren kontinuierlich angestiegen ist. ${ }^{15}$ Eine wissenschaftliche Aufbereitung der mit ähnlichen Problemen wie in Deutschland behafteten Aussetzungsdaten liegt nicht vor. Entnehmen lässt sich jedoch aus der Statistik, dass von den rund 7500 lifers immerhin gut 1100 eine Mindestverbüßungsdauer von mehr als 20 Jahren haben. Die Mindestverbüßungsdauer ist jedoch keinesfalls auch die tatsächliche Verbüßungsdauer: mehr als ein Drittel der einsitzenden Lebenslänglichen hatte diesen Zeitpunkt bereits überschritten. ${ }^{16}$

In den Niederlanden kann Mord mit lebenslanger oder einer zeitigen Freiheitsstrafe bestraft werden (Art. 289 Wetboek van Strafrecht). Weder für dieses noch für andere Verbrechen kennt das niederländische Gesetz jedoch eine zwingende Androhung lebenslanger Freiheitsstrafe, im Gegenteil: Immer ist auch ein zeitige Freiheitsstrafe (bis zu 30 Jahren) oder theoretisch sogar eine Geldstrafe als Hauptstrafe möglich. Seit 1950 wurde eine lebenslange Freiheitsstrafe insgesamt 55 Male ausgesprochen, in 12 Fällen allerdings von höheren Instanzen nicht bestätigt. ${ }^{17}$ Von den 43 bestandskräftigen Verurteilungen in 63 Jahren stammen über die Hälfte aus den letzten 13 Jahren. Beobachter machen eine Tendenz aus, wonach inzwischen - zumindest erstinstanzlich - auch einzelne Morde und nicht wie bisher nur Mehrfachmorde solchermaßen geahndet werden. ${ }^{18}$ Die Angaben über die Entlassungszahlen im genannten Zeitraum schwanken. Sicher ist jedoch, dass eine vorzeitige Entlassung ein sehr seltenes Ereignis ist und dass seit 1989 nur ein einziger dieser Gefangener entlassen wurde: Er war unheilbar krank und starb bald nach der Entlassung. ${ }^{19}$

In Spanien hingegen gibt es keine lebenslange Freiheitsstrafe. ${ }^{20}$ Sie gehörte traditionell nicht zum Sanktionsspektrum und wurde nach Abschaffung der Todesstrafe

14 Berücksichtigt man die Tatsache, dass es derzeit noch fast 6000 Inhaftierte mit der Sanktion der IPP gibt, sind rund $16 \%$ aller englischen und walisischen Gefangenen solche, die eine unbestimmte Freiheitsstrafe verbüßen.

15 Ministry of Justice, Offender Management Statistics Quarterly Bulletin October to December 2013 (www.gov.uk, zuletzt abgerufen am 2.5.2014), S. 8 und Vorausgaben.

16 Ministry of Justice: Table 1.4: Indeterminate sentence prisoner population, by tariff length and tariff expiry date (www.gov.uk/government/publications/offender-management-statistics-quarterly-2, abgerufen am 11.11.2013) sowie Ministry of Justice (Fn. 15).

17 www.rechtspraak.nl/Recht-In-Nederland/ThemaDossiers/levenslang/Pages/achtergrondinformatielevenslang.aspx (abgerufen am 21.11.2013).

18 W. Rutten, 'Lifelong Punishment: Equilibrium to Victims and Offenders?', Stichting Forum Levenslang, 2013, S. 19 (www.forumlevenslang.nl, abgerufen am 21.11.2013).

19 W. van Hattum, Levenslang, post Vinter', Nederlands Juristenblad (89) 2013, S. 1956 (1960).

20 Weitere europäische Strafgesetze sehen die lebenslange Freiheitsstrafe nicht als Sanktion vor, zumeist gibt es stattdessen relativ hohe zeitige Freiheitsstrafen (z. B. Kroatien mit bis zu 50 Jahren, niedriger liegt Portugal mit 25 Jahren, die auch bei Gesamtstrafenbildung nicht überschritten werden können). In manchen dieser Staaten gibt es Sanktionsformen, die der deutschen Maßregel der Sicherungsverwahrung entsprechen, z. B. in Norwegen, das mit 21 Jahren im Übrigen die im europaweiten Vergleich niedrigste zeitige Höchststrafe hat. Vgl. mit weiteren Beispielen EGMR, Vinter u.a../. Großbritannien (GK), (Fn. 1) Rn. 68. 
durch die Verfassung von 1978 auch nicht in den reformierten Código Penal (CP) von 1995 aufgenommen. ${ }^{21}$ Forderungen, eine lebenslange Freiheitsstrafe einzuführen, die besonders von Opferverbänden und in den Medien erhoben wurde, sind bislang nicht konkret aufgegriffen worden. ${ }^{22}$ Stattdessen sieht das spanische Strafgesetzbuch eine Höchstverbüßungsdauer von 40 Jahren Freiheitsstrafe vor (vor 2003 betrug sie 30 Jahre), die allerdings nicht für Einzeltaten, sondern für Mehrfachmorde, vor allem für Straftaten mit terroristischen Hintergrund in Betracht (Art. $76 \mathrm{CP}$ ) und de facto selten zu Anwendung kommt. ${ }^{23}$ Für Mord sind 15 bis 20 Jahren Freiheitsstrafe angedroht, 20 bis 25 Jahre, sofern mehrere Mordmerkmale verwirklicht sind (Art. 139, 140 CP). Eine zwingende Strafandrohung bei Mord gibt es damit nicht, der relative enge Strafrahmen lässt dem Tatgericht aber nicht viel Spielraum. Sowohl bei Ideal- als auch bei Realkonkurrenz nach deutschen Verständnis werden die Strafen im spanischen Urteil zunächst aufaddiert - im eingangs geschilderten Fall wurde für jeden Mord 29 Jahre, für jeden versuchten Mord 24 Jahre als Strafe (pena) verhängt. Zugleich wird die tatsächlich zu verbüßende Strafe (condena) festgelegt, im eingangs genannten Fall waren es 30 Jahre als in Frage kommendes Höchstmaß (Art. 70 Abs. 2 CP, Fassung zur Tatzeit). ${ }^{24}$

Die Statistik weist gemeinsam alle Gefangenen aus, die eine Freiheitsstrafe von 20 Jahren und mehr verbüßen. Dies waren zum 1.9.2011 1412 Personen - knapp 2\% aller Gefangenen bzw. 2,3\% aller Strafgefangenen. ${ }^{25}$ Von ihnen dürften nur wenige, etwa die Attentäter der terroristischen Attacken von Madrid 2004 sowie ETA-Terroristen, zu der hier besonders interessierenden Kategorie derjenigen zählen, die eine Strafe von 30 oder mehr Jahren (potenziell) voll verbüßen müssen, es wird von etwa

21 J. L. de la Cuesta/I. Blanco, Spain, in: D. van Zyl Smit/F. Dünkel (Hrsg.), Imprisonment today and tomorrow, 2. Aufl., Den Haag u.a. 2001, S. 609 (609f.).

22 J. Cid, J./B. Tébar, Spain, in: N. Padfield/D. van Zyl Smit/F. Dünkel, (Hrsg.), Release from Prison. European Policy and Practice, Cullompton 2010, S. 358 (368).

23 Ich bedanke mich bei Prof. Josep Cid, Barcelona, für Hintergrundinformationen. Zuletzt wurde in einem Mordfall, in den spanischen Medien besonders viel Beachtung fand, ein Mann wegen des Mordes an seinen beiden Kindern zu insgesamt 40 Jahren verurteilt, vgl. El Pais vom 22.7.2013. Der Richter setzte fest, dass die Lockerung, die der Strafaussetzung vorangehen muss, frühestens nach 20 Jahren in Betracht kommt.

24 Nach der spanischen Strafprozessordnung können bei ausreichendem Zusammenhang zwischen Tatkomplexen Strafen für die Vollstreckung zusammengezogen werden, auch wenn sie aus verschiedenen Urteilen stammen ( $\$ 988$ Ley de Enjuiciamiento Criminal). Hier kann ebenfalls nicht über die geltende Höchstverbüßungsdauer hinausgegangen werden.

25 Quelle: Eigene Berechnungen nach Daten des Europarats, M. Aebi, N. /Delgrande, N. Council of Europe Annual Penal Statistics; SPACE I, Survey 2011, PC-CP (2013) 5, Straßburg 2013 (http://www3.unil.ch/wpmu/space/, abgerufen 20.11.2013, an gleicher Stelle sind auch die Surveys der Jahre 1999 bis $2010 \mathrm{zu}$ finden). In der Untersuchung von Tébar lagen die Zahlen höher (B. Tébar, La aplicación de la libertad condicional en España, Revista de derecho penal y criminologia [18] 2006, S. $283[296])$. 
bis zu 60 betroffenen ETA-Gefangenen gesprochen. ${ }^{26}$ Aus einer stichtagsbezogenen regionalen Vollerhebung in Katalonien im Jahr 2003, die zum Untersuchungszeitpunkt 3/4 ihrer Strafe verbüßt hatten und damit prinzipiell entlassungsberechtigt waren, geht ferner hervor, dass bei denjenigen, die zwischen 25 und 30 Jahren (das zum Untersuchungszeitpunkt geltende Höchstmaß) zu verbüßen hatten, immerhin auch über die Hälfte vorzeitig entlassen wurde..$^{27}$

Im letzten Eingangsbeispiel schließlich waren neben der Mordanklage Verbrechen gegen die Menschlichkeit Gegenstand des Verfahrens. Anders als nationale Strafgesetzbücher sieht das Statut des Internationalen Strafgerichtshofs für das ehemalige Jugoslawien ${ }^{28}$ keinen Strafrahmen für einzelne Delikte vor. Nach Art. 24 des Statuts und Nr. 101 der Verfahrensordnung sollen die Strafkammern bei der Festsetzung der Strafe Umstände wie die Schwere der Straftat und die persönlichen Verhältnisse des Verurteilten in Betracht ziehen. Im Hinblick auf deren Länge sollte sich der Gerichtshof an der allgemeinen Praxis im ehemaligen Jugoslawien orientieren. Das war insofern schwierig, als das jugoslawische Strafgesetzbuch zwar die Todesstrafe vorsah, die lebenslange Freiheitsstrafe aber 1959 in einer Reform, die das Strafrecht tendenziell liberalisierte, abgeschafft worden war, und die höchste zeitige Freiheitsstrafe 20 Jahre betrug. ${ }^{29}$ Tatsächlich hat sich der Gerichtshof in seiner Verfahrensordnung die Befugnis verliehen, lebenslange Freiheitsstrafen zu verhängen und hat auch keine Höchstgrenze für zeitige Freiheitsstrafen festgelegt. ${ }^{30}$ Eine zwingende Androhung lebenslanger Freiheitsstrafen gibt es jedoch nicht. Bislang sind 74 Verfahren rechtskräftig mit einer Verurteilung abgeschlossen (Stand 2.5.2014). Zwei Verfahren endeten mit einer lebenslangen Freiheitsstrafe, die höchste zeitige Freiheitsstrafe, die in der zweiten Instanz bestätigt wurde, waren 40 Jahre (zwei Fälle),

26 J.-L. de la Cuesta Arzamendi, Strengthening peace and the memory of the victims of terrorism: efforts and perspectives in the Basque Country, Vortrag bei der Jahrestagung der European Society of Criminology 2012, Bilbao (unveröff. Manuskript). Ein Anhaltspunkt ergibt sich aus allein 28 Verfassungsbeschwerden im Zusammenhang der doctrina parot, d. h. der Rechtsprechungsänderung, die eine vorzeitige Entlassung bei Terroristen praktisch versagt, vgl. (EGMR [GK] Del Rio Prada./. Spain, [Fn. 1], Rn. 31).

27 Tébar, Libertad condicional (Fn. 25), S. 305.

28 Verabschiedet am 25. Mai 1993 durch Resolution 827 des Sicherheitsrates, zuletzt geändert am 7.7.2009 durch Resolution 1877 des Sicherheitsrats, auf Englisch abrufbar unter www.icty.org. Eine deutsche Fassung (Stand 2002) ist dem Gesetz über die Zusammenarbeit mit dem Internationalen Strafgerichtshof für das ehemalige Jugoslawien vom 10.4.1995, BGBl. I, S. 495 als Anhang beigefügt.

29 D. Petrovec/A. Selih, Slowenien, in: F. Dünkel et al. (Hrsg.) Kriminalität, Kriminalpolitik (Fn. 9), S. $783(785)$.

$30 \mathrm{Nr} .101$ (A) der Verfahrensordnung und Beweisregeln des Internationalen Strafgerichtshofs für das ehemalige Jugoslawien vom 11.2.1994, zuletzt geändert am 22.5.2013, abrufbar unter www.icty.org. Kritisch zu diesem wenig prinzipiengeleiteten Vorgehen D. van Zyl Smit, Aspekte der Strafzumessung in der internationalen Strafjustiz, ZStW (115) 2003, S. 931 (941 ff.). Sehr kritisch mit Blick auf die konkrete Strafzumessung plädiert Hoven für eine obligatorische lebenslange Freiheitstrafe für die meisten der an den internationalen Strafgerichten abgeurteilten Delikte. (E. Hoven, Elf Stunden für ein Menschenleben - Zur Strafzumessung im Völkerstrafrecht, ZStW [125] 2013, S. 137 [168]). 
zwei Verfahren endeten mit einer Freiheitsstrafe von 35 Jahren, je einmal mit 29 und 27 Jahren. $^{31}$

\section{Strafzwecke bei der lebenslangen und den sehr langen zeitigen Freiheitsstrafen}

Der EGMR musste sich mit dem Problem legitimer Strafzwecke auseinandersetzen; insbesondere also, inwieweit neben den spezialpräventiven auch Vergeltungszwecke eine Freiheitsstrafe, die bis zum Tode vollstreckt werden soll, vor Art. 3 EMRK rechtfertigen können. Ohne eine erschöpfende vergleichende Strafzweckdiskussion anzustreben, sollen deshalb im Folgenden augenfällige Unterschiede zwischen den nach Dogmatik, Gesetz und Rechtsprechungspraxis bemühten Strafzwecken umrissen werden. Die untersuchten Rechtsordnungen differenzieren dabei teilweise zwischen allgemeinen Kriterien und denen für die lebenslange Freiheitsstrafe. Mitunter wird dies über verschiedene Aussetzungsmodalitäten getan, deshalb sind beide Aspekte - Strafzwecke (II. 2) und verschärfte Entlassungsvoraussetzungen (II. 3) - in der Darstellung nicht klar zu trennen.

Das deutsche Recht weist für die lebenslange Freiheitsstrafe zunächst keine Strafzweckbesonderheiten auf, $\mathbb{4} 46 \mathrm{StGB}$ gilt für diese Sanktion ebenso wie für alle anderen. Grundlage der Strafzumessung ist die Schuld des Täters, wobei hier Tat und Täter zu betrachten sind. Die Tat bzw. das Gewicht der durch sie bewirkten Rechtsverletzung ist damit der wesentliche Anknüpfungspunkt für die Strafzumessung. ${ }^{32}$ Ebenso sind aber „die Wirkungen, die von der Strafe für das zukünftige Leben des Täters“ zu erwarten sind, bei der Strafzumessung zu berücksichtigen. Damit wird der Möglichkeit der Resozialisierung des Täters durch Strafvollstreckung bzw. Strafvollzug besonders große Bedeutung zugewiesen. ${ }^{33}$ Prinzipiell stellen Resozialisierung, Prävention, Schuldausgleich, Sühne und Vergeltung für begangenes Unrecht gleichermaßen verfassungsgemäße Aspekte einer angemessenen Strafsanktion dar. ${ }^{34}$

31 Quelle: http://www.icty.org/sections/TheCases/KeyFiguresoftheCases, abgerufen am 2.5.2014. In einem Verfahren waren in der ersten Instanz 45 Jahre verhängt worden, der Schuldspruch und damit das Strafmaß hatten aber nicht in vollem Umfang Bestand. Derzeit (Stand 2.5.2014) sind erstinstanzlich in drei Fällen, die die Massaker von Srebrenica betreffen, ebenfalls lebenslange Freiheitsstrafen verhängt worden (Popović und Beara, beide IT-05-88; Tolimir IT-05-88/2).

32 W. Stree/J. Kinzig, in: A. Schönke,Schröder (Hrsg.), StGB, 28. Auflage, München 2010\$46 Rn. 4; F. Streng, Strafrechtliche Sanktionen, 3. Aufl., Stuttgart 2012, S. 255 ff., jew. mit vielen Nachweisen zu diesen stark umstrittenen Problemen.

33 Das ist eine Entwicklung der 1960er Jahre: Bei den Beratungen zur Reform des Strafgesetzbuches (StGB) E 1962 (BTDrucks IV/650, S. 204), hieß es zur vorzeitigen Entlassung noch, sie erhielte ,ihre Rechtfertigung aus dem Besserungszweck der Strafe. Lebenslängliche Freiheitsstrafen dienen aber ihrer Natur nach nicht diesem Zweck, sondern ausschließlich oder mindestens ganz überwiegend dem Ausgleich der Schuld, die der Täter auf sich geladen hat. Ihr Vollzug hat nicht den Sinn der Vorbereitung auf ein späteres Leben in der Freiheit.".

34 Verfassungsrechtlich gefordert ist ein „sinn- und maßvolles Strafen“, vgl. BVerfGE 28, 368 (391), bestätigt auch in BVerfGE 45, 187 (254), wo aber davon ausgegangen wird, dass „im Wesentlichen der herrschenden Vereinigungstheorie entsprechenden Strafzwecke“ zu berücksichtigen sind. 
Bei der Erörterung der Frage, ob mit einer lebenslangen Freiheitsstrafe legitime Strafzwecke verfolgt werden können, diente dem Bundesverfassungsgerichts bei seiner maßgeblichen Entscheidung aus dem Jahr 1977 die Generalprävention als Ausgangspunkt, weil es davon ausging, dass sie „als Sanktion für schwerste Tötungsdelikte zum Schutz des menschlichen Lebens als eines überragenden Rechtsguts eine wichtige Funktion erfüllt, den heute im Volke lebenden Wertvorstellungen entspricht und gleichzeitig ein bewußtseinsbildendes Unwertsurteil verdeutlicht". ${ }^{35}$ Seit dem „Lebach-Urteil“36 des Gerichts vier Jahre zuvor ist jedoch anerkannt, dass aus Art. 2 Abs. 1 und Art. 1 Abs. 1 GG in Verbindung mit dem Sozialstaatsprinzip ein grundgesetzlich verbürgter Anspruch des Verurteilten auf Resozialisierung erwächst. Dennoch: „Hält der Gesetzgeber die Sühne weiterhin für einen legitimen Strafzweck, so kann er sich davon leiten lassen, daß der Straftäter mit der Vernichtung eines menschlichen Lebens durch Mord schwerste Schuld auf sich geladen hat und seine Wiedereingliederung in die Rechtsgemeinschaft eine Schuldverarbeitung voraussetzt, die auch durch eine sehr lange Freiheitsstrafe mit der Chance vorzeitiger Entlassung ermöglicht wird.“

Die Konsequenz aus dieser Feststellung ist die Verrechtlichung der „Chance vorzeitiger Entlassung “ in $\$ 57$ a StGB. Eine Besonderheit ist bei manchen Verurteilungen zu lebenslanger Freiheitsstrafe die Feststellung der „besonderen Schwere der Schuld“ nach $\mathbb{5} 57 \mathrm{a}$ Abs. 1 S. 1 Nr. 2 StGB. Vieles ist hier umstritten. ${ }^{37}$ Letztlich ist die Schuldschwereklausel des $\$ 57$ a StGB die Konsequenz aus der zwingenden Strafandrohung des $\mathbb{2} 211 \mathrm{StGB}$, denn nur über die Entlassungspraxis nach dieser Vorschrift ist bei Mord, der in nahezu allen Fällen der Verhängung lebenslanger Freiheitsstrafen zugrunde liegt, überhaupt eine Differenzierung nach der Schuldschwere möglich. Daher besteht weitestgehend Einigkeit darüber, dass mit dieser Schuld die Strafzumessungsschuld des $\mathbb{} 46$ Abs. 1 S. 1 StGB gemeint ist, d. h. dass es um den besonderen Unrechtsgehalt der Tat geht und allgemeine generalpräventive Aspekte zu ihrer Begründung nicht herangezogen werden dürfen. Unzulässig wäre zum Beispiel die Argumentation, das Rechtsbewusstsein der Bevölkerung würde empfindlich gestört, wenn im konkreten Fall nicht auf die besondere Schwere der Schuld erkannt werden würde (etwa bei einem besonders spektakulären Fall, einem bekannten Opfer, besonderem Medieninteresse o. ä.). ${ }^{38}$ Das Bundesverfassungsgericht erklärte die Schuldschwereklausel unter der Maßgabe für verfassungsgemäß, dass schon das

37 Vgl. hierzu z. B. F. Dünkel, in: U. Kindhäuser/U. Neumann/U. Paeffgen, Nomos Kommentar Strafgesetzbuch, 3. Auflage, Baden-Baden 2013, $\$ 57$ a Rn. 7 ff.; und Kett-Straub, Lebenslange Freiheitsstrafe, (Fn. 3), S. $212 \mathrm{ff}$.

38 Dünkel, NK-StGB (Fn. 37), \57a Rn. 8; Streng, NK-StGB (Fn. 37), \46 Rn. 161; näher zum Ganzen und zur „Reflexwirkung“ der Generalprävention im Sinne eines funktional geprägten Schuldbegriffs Kett-Straub Lebenslange Freiheitsstrafe, (Fn. 3), S. 233 ff. und 283. 
Tatgericht zum Urteilszeitpunkt über die Frage, ob eine besondere Schwere der Schuld vorliegt, entscheidet. ${ }^{39}$ Andererseits ist die Klärung der Frage, ob und wie lange die Schwere der Schuld nun eine längere Vollstreckung gebietet, Sache der Strafvollstreckungskammer und muss mit erheblichem zeitlichem Abstand zur Aburteilung entschieden werden. Dass an dieser Stelle weitere Faktoren und letztlich auch andere Strafzwecke als der Schuldausgleich herangezogen werden, ist nicht verwunderlich und wird von manchen als "Strafzumessungsbrei“ ${ }^{40}$ kritisiert. Beispiele für solche anderen Gründe sind humanitäre Erwägungen wie das hohe Alter des Betroffenen oder der schlechte Gesundheitszustand, aber auch eine positive Persönlichkeitsentwicklung oder besondere Resozialisierungsbemühungen, mithin spezialpräventiv relevante Faktoren. ${ }^{41}$

In England und Wales wird bei der lebenslangen Freiheitsstrafe zwischen dem punitiven Element und einem präventiven Element, das von der Gefährlichkeit des Verurteilten abhängt, unterschieden. Für das bis 2003 geltende Recht ging das House of Lords in seiner Eigenschaft als höchstes Gericht davon aus, dass das punitive Element den zwingend zu verbüßenden Zeitraum (tariff) bestimmt, es müsse sich an der Natur der Tat orientieren. Die Richter waren in früheren Entscheidungen zurückhaltend $^{42}$ gewesen und hatten angedeutet, dass im Einklang mit der EMRK nur eine auch spezialpräventiv (d. h. durch Gefährlichkeit) begründete Freiheitsstrafe bis zum Tode vollstreckbar sei. Eine Trendwende vollzogen sie spätestens im spektakulären Fall Hindley, die mit ihren Partner mindestens fünf Kinder gequält und umgebracht hatte. Sie stellten fest, dass es keinen Grund gäbe, „warum ein oder mehrere Verbrechen, soweit sie ausreichend abscheulich („heinous“) sind, nicht eine lebenslange Inhaftierung zum Zwecke reiner Bestrafung verdienen (Übers. der Verf.)“ Die Richter bezogen sich dabei auf die Gesetzesmaterialien für den erwähnten Murder (Abolition of the Death Penalty) Act. ${ }^{43}$ Auch das Konzept des tariff zwinge dann nicht dazu, von einer bis zum Tode vollzogenen Freiheitsstrafe abzusehen, wenn die Bedürfnisse nach Vergeltung und Abschreckung (,,requirements of retribution and deterrence“) anders nicht befriedigt werden könnten. Später wurde dies bekräftigt: Prinzipiell seien legitime Strafzwecke „Bestrafung“ („punishment“, im Deutschen würde man wohl den Begriff Vergeltung wählen), Abschreckung, Wiedereingliede-

39 BVerfGE 86, 288 (Ls. 3a) unter Hinweis auf BVerfGE 72, 105 (114).

40 Diese Formulierung stammt von den Autoren des Alternativ-Entwurf Leben, GA 2008, S. 193 (270).

41 Näher und mit weiteren Nachweisen Kett-Straub, Lebenslange Freiheitsstrafe (Fn. 3), S. 217 und $277 \mathrm{ff}$.

42 In R v. Lichniak and $R v$. Pyrah wurde die Vereinbarkeit der obligatorischen Freiheitsstrafe mit Art. 3 EMRK gerade damit begründet, dass allein spezialpräventive Gründe darüber bestimmten, ob über den festgesetzten tariff hinaus eine lebenslange Freiheitsstrafe verbüßt werden muss, ungefährliche Täter also eine reelle Chance hätten, wieder in Freiheit zu gelangen.

43 Regina v. Secretary of State for the Home Department, ex parte Hindley, Entscheidung vom 30. März 2000 (www.publications.parliament.uk/). 
rung (rehabilitation) und Schutz der Bevölkerung. ${ }^{44}$ Wenn die Schwere der Tat auBergewöhnlich sei, rechtfertigten die Strafzwecke der Vergeltung und Abschreckung auch die Verhängung einer bis zum Tode vollziehbaren Freiheitsstrafe.

In den Niederlanden findet sich im Strafgesetzbuch keine Aussage zu den Strafzwecken. Den verschiedenen Sanktionen des niederländischen Strafgesetzbuchs liegen jedoch - in Theorie und Praxis - verschiedene Strafzwecke zugrunde, je nach Sanktion Vergeltung, negative Spezial und Generalprävention, Resozialisierung, Konfliktregelung, Schutz der Öffentlichkeit und Wiedergutmachung. In der Praxis ist die Tatschuld Anknüpfungspunkt. In der Regel werden Strafzwecke im Urteil nicht thematisiert, oft kann aber aus der Wahl der Sanktion oder der Kombination von Sanktionen auf die angestrebten Zwecke der Strafe im konkreten Fall zurückgeschlossen werden. ${ }^{45}$ Als der damals für das Amt des Ministerpräsidenten kandidierende Politiker Fortuyn, der durch islamfeindliche und weitere extreme politische Positionen bekannt geworden war, ermordet wurde, schockierte dies die niederländische Gesellschaft heftig. Dennoch wurde der Täter, ein militanter Umwelt- und Tierschutzaktivist, nicht zu lebenslanger Freiheitsstrafe verurteilt, sondern zu 18 Jahren Freiheitsstrafe. Beide befassten Instanzen setzten sich im Rahmen der Strafzumessungsüberlegungen nicht nur mit der Schwere des Delikts, sondern auch mit der heftigen Erregung der Öffentlichkeit und dem „nicht wiedergutzumachenden Schaden für den demokratischen Prozess" 46 auseinander. Dennoch kamen sie zu dem Schluss, dass der Verurteilte „aus humanitären Gründen“ eine Chance auf die Rückkehr in die Gesellschaft haben müsse bzw. dass bei einem Ersttäter trotz der Schwere der Tat und den besonderen Begleitumständen eine lebenslange Freiheitsstrafe nicht angemessen sei. Einige Jahre später hingegen wurde in dem einleitend verwendeten Fall wieder eine umstrittene Person des öffentlichen Lebens, ein islamkritischer Regisseur, ermordet. Die Wirkung auf die niederländische Gesellschaft war vergleichbar und diesmal wurde der Täter zu lebenslanger Haft verurteilt - das Gericht wertete zu seinen Lasten, dass er keinerlei Bedauern über die Tat zeigte und eine Rückfälligkeit wahrscheinlicher sei. ${ }^{47}$ Damit wird im Falle der lebenslangen Freiheitsstrafe deutlich, dass es zum einen um Vergeltung, zum anderen aber auch um Spezialprävention in

44 Regina $v$. Bieber, [2008] EWCA Crim 1601. Ähnliche Ausführungen finden sich auch in Regina $v$. Oakes u.a., [2012] EWCA Crim 2435 (die Entscheidungen sind abrufbar in der Datenbank www.judiciary.gov.uk).

45 P. Tak, P., The Dutch criminal justice system, Nijmegen 2008, S. 130 ff.; M. Boone/M. Moerings, Niederlande, in: F. Dünkel et al. (Hrsg.) Kriminalität, Kriminalpolitik (Fn. 9), S. 666 (666 ff.).

46 Berufungsgericht Amsterdam, Urteil vom 18. Juli 2003, zu finden als NJ 2003, 580 in der niederländischen Rechtsprechungsdatenbank www.rechtspraak.nl.

47 Distriktgericht Amsterdam, Urteil vom 26. Juli 2005, zu finden als LJN AU0025 580 in der niederländischen Rechtsprechungsdatenbank www.rechtspraak.nl. 
Form der incapacitation des Täters geht. ${ }^{48}$ Resozialisierungsaspekte hingegen sind irrelevant.

In Spanien ist im Zuge der Reform des Strafgesetzbuchs die Vereinigungstheorie umgesetzt, die der Vorgabe des Art. 25 Abs. 2 der spanischen Verfassung folgt, nach der freiheitsentziehende Strafen auf Erziehung und Resozialisierung (reeducación y reinserción social) gerichtet sein müssen. ${ }^{49}$ Die Literatur der 1990er Jahre ging zumindest teilweise davon aus, dass vor diesem Hintergrund lebenslange und auch sehr lange Freiheitsstrafen verfassungsrechtlich nicht haltbar sind. Schon bei Freiheitsstrafen mit einer tatsächlichen Vollzugsdauer über 15 Jahren wurde unter Berufung auf strafvollzugswissenschaftliche Erkenntnisse davon ausgegangen, dass sie die Persönlichkeit irreparabel schädigen könnten. Kritisiert wurde die Möglichkeit, die volle Höchstdauer von damals 30 Jahren als Verbüßungszeit auszuschöpfen, ${ }^{50}$ wie dies auch im eingangs skizzierten Fall geschehen ist. Diese Liberalität der Transitionsjahre ist auch in Spanien punitiveren kriminalpolitischen Bestrebungen ${ }^{51}$ gewichen - wie bereits erwähnt, kann sich nach der konservativen Strafrechtsreform von 2003 die vollstreckbare Freiheitsstrafe inzwischen auf 40 Jahre belaufen.

In Bezug auf die internationale Strafgerichtsbarkeit ist über Strafzwecke viel gestritten worden. ${ }^{52}$ In den Urteilen des Jugoslawien-Gerichtshofs finden sich verschiedene Erwägungen - genannt werden Abschreckung, Vergeltung, incapacitation, Resozialisierung, ${ }^{53}$ als spezifischer Strafzweck auch der Beitrag des Urteils zur Konfliktbeendigung. ${ }^{54}$ Nach inzwischen gefestigter Rechtsprechung des Gerichts sind „primary objectives of sentencing... punishment and deterrence, " 55 wobei im Mittelpunkt die Vergeltung steht. ${ }^{56}$ Eine tatbestandsorientierte Differenzierung bzw. tatproportionale Strafzumessung angesichts der vor den internationalen Strafgerichtshöfen abgeurteilten Art von Taten ist schwierig. Im hier gewählten Beispiel hatte die zuständige Kammer des Jugoslawien-Gerichtshofs die Schuld des Angeklagten zum allein relevanten Maßstab gemacht, sie wiege so schwer, dass die vorgetragenen schuld-

48 Urteil des Hooge Rat, des obersten Gerichtshofs der Niederlande, vom 14.3.2006, zu finden als LJNAU 5496 in der niederländischen Rechtsprechungsdatenbank www.rechtspraak.nl.

49 J. Cerezo Mir, Das neue spanische Strafgesetzbuch von 1995, ZStW (108) 1996, S. 858.

50 J. Cerezo Mir, Spanien (Fn. 49), S. 859 m. w. N.

51 Einen lesenswerten Überblick zur kriminalpolitischen Entwicklung in dieser Hinsicht geben E. Larrauri/ J. Cid, Spanien, in: F. Dünkel et al. (Hrsg.) Kriminalität, Kriminalpolitik (Fn. 9), S. 820 (820 ff.).

52 Vgl. z. B. die Zusammenfassung in Hoven, Strafzumessung (Fn. 30), S. 137 ff., auch van Zyl Smit, Strafzumessung (Fn. 30), S. 945 ff.

53 Sie wird jedoch als von untergeordneter Bedeutung betrachtet, z. B. Prosecutor v. Blasic', IT-95-14-T, Trial Chamber, 3 March 2000, Rn. 782.

54 Z. B. Prosecutor v. Blasic (Fn. 53) Rn. 762; Prosecutor v. Milan Lukić and Sredoje Lukić, Case No. IT-98-32/1-A, Trial Chamber, 20 December 2009, Rn. 1049.

55 Seit Prosecutor v. Jelisić, IT-95-10, Trial Chamber, 14 December 1999, Rn. 116; hier Prosecutor v. Milan Lukić and Sredoje Lukić Rn. 1049.

56 Prosecutor v. Aleksovski, IT-95-14/1-A, Appeals Chamber, 24 March 2000, Rn. 182; van Zyl Smit, Strafzumessung (Fn. 30), S. 944. 
mindernden Gründe (z. B. das relativ geringe Alter zum Tatzeitpunkt oder die Tatsache, dass er zuvor unbescholten war) von zu geringem Gewicht seien. Die Schwere der Schuld wird mit der Opferzahl von mindestens 138 Menschen und Beschreibungen der Tat bzw. der Persönlichkeit des Angeklagten als „kaltblütig“ und geprägt von „schierer Brutalität“ unterlegt. ${ }^{57}$ Soweit ersichtlich, kommt der Aspekt der Resozialisierung nur noch abstrakt vor - prinzipiell muss bei der Würdigung der Person bzw. des Charakters des Angeklagten auch seine Wiedereingliederungsfähigkeit („propensity for rehabilitation“) $)^{58}$ in Betracht gezogen werden - eine Überlegung, wie sich eine lebenslange Freiheitsstrafe konkret auswirkt, fehlt jedoch im betreffenden Urteil.

\section{Strafrestaussetzung bei lebenslangen Freiheitsstrafen und ihre Begründung}

Die Vereinbarkeit der lebenslangen Freiheitsstrafe mit Grund- und Menschenrechten hängt nach dem Gesagten mit der Möglichkeit der Entlassung aus dem Gefängnis zu Lebzeiten zusammen. In Deutschland war es bis zur Einführung des $\$ 57$ a StGB im Jahr 1981 allein dem Gnadenträger vorbehalten, für eine vorzeitige Entlassung von zu lebenslanger Freiheitsstrafe Verurteilten zu sorgen. De facto wurden nach 1965 wohl die meisten dieser Verurteilten ${ }^{59}$ vorzeitig entlassen: Nach einer Erhebung lag der Median der Verbüßungszeit zwischen 1945-1975 bei gut 20 Jahren, entscheidend war letztlich immer eine positive Sozialprognose. ${ }^{60}$ Dennoch verurteilte das Bundesverfassungsgericht bei grundsätzlicher Akzeptanz der lebenslangen Freiheitsstrafe die Gnadenpraxis als verfassungswidrig. In der Begründung wurden die verfassungsrechtlichen Grenzen der Gestaltungsfreiheit des Gesetzgebers, die lebenslange Freiheitsstrafe bei schwersten Delikten beizubehalten, aus Art. 1 GG abgeleitet. ${ }^{61}$ Niemand dürfe zum „bloßen Objekt der Verbrechensbekämpfung unter Verletzung seines ... sozialen Wert- und Achtungsanspruchs gemacht werden “. ${ }^{62}$ Das Bundesverfassungsgericht prüfte mit einem beachtlichen Aufwand an externer Expertise ${ }^{63}$ die Frage, ob und wann irreparable Haftschäden aufträten, die die lebenslange Freiheitsstrafe per se eine mit diesem Verständnis des Art. 1 GG nicht zu vereinbarende Strafe machen würde, kam in dieser Frage aber zu keinem eindeutigen Schluss. ${ }^{64}$

57 Prosecutor v. Milan Lukić and Sredoje Lukić, Case No. IT-98-32/1-A, Appeal Chamber, 4 December 2012, Rn. 649.

58 Prosecutor v. Milan Lukić and Sredoje Lukić (Fn. 57), Rn. 1056.

59 K. Laubenthal, Lebenslange Freiheitsstrafe: Vollzug und Aussetzung des Strafrestes, S. 106 (Fn. 11).

60 BVerfGE 45, 187 (203 f., 218 ff.).

61 BVerfGE 45, 187 (227ff.).

62 BVerfGE 26, 389 (391) und wiederholt auch in BVerfGE 45, 187 (227).

63 Vgl. zusammenfassend Kett-Straub, Lebenslange Freiheitsstrafe (Fn. 3), S. 48 ff. Alle Gutachten sind abgedruckt bei H.-H. Jescheck/O. Triffterer (Hrsg.), Ist die lebenslange Freiheitsstrafe verfassungswidrig? - Dokumentation über die mündliche Verhandlung vor dem Bundesverfassungsgericht am 22. und 23. März 1977, Baden-Baden 1978. 
Nach der Bewertung des Gerichts (und mit Zustimmung aus der Literatur) ${ }^{65}$ ergibt sich aber aus der Menschenwürdegarantie im Zusammenspiel mit dem Anspruch auf Resozialisierung (aus Sozialstaatsprinzip, Art. 1 und Art. 2 GG) ${ }^{66}$ zwingend die Konsequenz, dass jeder zu einer lebenslangen Freiheitsstrafe verurteilte Gefangene sowohl Anspruch auf resozialisierungsfördernde Behandlung im Vollzug als auch eine „konkrete und grundsätzlich auch realisierbare Aussicht“ auf Entlassung haben muss. ${ }^{67}$

Für den hier angestellten Vergleich zwischen verschiedenen Rechtsordnungen ist die Passage des Urteils am wichtigsten, in der das Gericht formuliert „... der Kern der Menschenwürde wird getroffen, wenn der Verurteilte ungeachtet der Entwicklung seiner Persönlichkeit jegliche Hoffnung, seine Freiheit wiederzuerlangen, aufgeben muß.“68

Das Gericht bezog dann in einem zweiten Schritt auch das Rechtsstaatsprinzip in seine Argumentation ein und widmet sich der prozessualen Absicherung: Dabei ging es davon aus, dass trotz der geübten Sorgfalt Entlassungsentscheidungen „schlechterdings nicht Sache des Gnadenträgers“ sein können. Allerdings nimmt es nicht selbst Stellung zur Frage, ob es notwendigerweise Sache eines Gerichts sein müsse, Art. 104 GG kommt in der Argumentation nicht vor. Das Gericht geht auch nicht ausdrücklich auf die Gefahren politischer und medialer Einflussnahme auf die Gnadenträger ein, sondern begründet die Notwendigkeit, das anzuwendende Verfahren gesetzlich zu regeln, allgemein mit dem Bedarf an justizförmiger Garantien. Gegnern einer gesetzlichen Fixierung der Aussetzungsmöglichkeit, die insbesondere davon ausgingen, dass sie mit dem Schuldgrundsatz nicht in Einklang zu bringen seien, wird unter Verweis auf Parallelen zu den zeitigen Freiheitsstrafen widersprochen. ${ }^{69} \mathrm{Geg}$ nern, die eine Erosion der Strafe bzw. ihrer abschreckenden Wirkung befürchteten, wird entgegengehalten, dass die Bevölkerung schon an die Entlassungspraxis auf dem Gnadenwege gewöhnt sei. Letztlich wird das Gebot, das Verfahren gesetzlich zu bestimmen, direkt aus der Schwere des Grundrechtseingriffs bzw. der „existentiellen Bedeutung “ der Entscheidung abgeleitet. ${ }^{70}$

Als Reaktion auf dieses und weitere Urteile ${ }^{71}$ werden in Deutschland die Aussetzungsentscheidungen gem. $\$ 57$ a StGB durch die Vollstreckungskammern getroffen

65 Vgl. z. B. Kett-Straub, Lebenslange Freiheitsstrafe (Fn. 3), S. 46; Dünkel, NK-StGB (Fn. 37), \$57a StGB Rn. 1; H. Müller-Dietz, Schuldschwere und Urlaub aus der Haft, JR 1984, S. 353 (361).

66 BVerfGE 45, 187 (238ff.) unter Verweis auf BVerfGE 35, 202 (235 f.) - Lebach.

67 BVerfGE 45, 187 (245 f.), vgl. auch Dünkel, NK-StGB (Fn. 37), \$57a StGB Rn. 1.

68 BVerfGE 45, 187 (245f.).

69 Vgl. hierzu auch Kett-Straub, Lebenslange Freiheitsstrafe (Fn. 3), S. 98 f.

70 BVerfGE 45, 187 (245 f.).

71 Ein Überblick zu den verfassungsrechtlichen Problemen findet sich bei Dünkel, NK-StGB (Fn. 37), \57a Rn. 1 und 7, eine ausführliche Darstellung bei Kett-Straub, Lebenslange Freiheitsstrafe (Fn. 3), S. $102 \mathrm{ff}$. 
( $\$ 454$ StPO). Entscheidend für die Aussetzung des Strafrests zur Bewährung nach der Mindestverbüßungszeit von 15 Jahren ist eine günstige Gefährlichkeitsprognose - die Entlassung muss „unter Berücksichtigung des Sicherheitsinteresses der Allgemeinheit verantwortet“ ( $\$ 57$ a Abs. 1 . Nr. 3 i.V.m. $\$ 57$ Abs. 1 . Nr. 2 StGB) werden können. Liegen diese Prognose und die übrigen Aussetzungsvoraussetzungen vor, muss das Gericht aussetzen, ein Ermessen steht ihm nicht zu. In den hier besonders interessierenden Fällen besonderer Schuldschwere muss als besonderes Merkmal geprüft werden, ob sie eine weitere Vollstreckung (d.h. über 15 Jahre hinaus) „gebietet". Wie dieser unbestimmte Rechtsbegriff konkret auszufüllen ist, ist umstritten und führt letztlich zu der oben schon beschriebenen zweiten Prüfung. ${ }^{72}$ Dabei ist die Deutung, wonach ihm eine Begrenzungsfunktion ${ }^{73}$ zukommt und er nicht eine Regelvermutung zugunsten einer deutlichen Verlängerung der Mindestverbüßungsdauer aufstellt, überzeugend (allerdings in der Praxis nicht herrschend). Die Schwere der Schuld kann die Verbüßungszeit im Ergebnis deutlich verlängern. ${ }^{74}$

In England und Wales sind, wie oben beschrieben, zwei verschiedene Formen der lebenslangen Freiheitsstrafe ${ }^{75}$ denkbar - solche, die eine Aussetzung nach Verbüßung einer bestimmten Mindestdauer prinzipiell ermöglichen und der Sonderfall, in dem die Verbüßungsdauer nicht begrenzt bzw. bis zum Tode festgesetzt wird. Die Mindestverbüßungsdauer (tariff) wird seit 2003 der Neuregelung durch den CJA von 2003 vom Gericht (sentencing court) zusammen mit dem Strafausspruch festgesetzt. Zuvor war es der Innenminister (Secretary of State), der sie bestimmte und stets nach 25 Jahren überprüfte, ob eine Weitervollstreckung geboten war oder der Betreffende doch bedingt entlassen werden sollte. ${ }^{76}$ Interessanterweise war es eine höchstrichterliche Entscheidung, die zu diesem Zuständigkeitswechsel führte, weil die bisherige Regelung, nach der ein Exekutivorgan über die effektive Mindestlänge der Strafe entschied, nach Auffassung der Richter gegen die prozessualen Garantien des Art. 6 EMRK verstieß.77 Allerdings fiel mit der Zuständigkeitsverschiebung die Überprüfung nach 25 Jahren ebenfalls fort - zwar kann die Festsetzung der whole life order sogleich im normalen Rechtsweg angefochten werden, eine weitere Überprüfung während der Vollstreckungszeit gibt es jedoch nicht mehr.

72 Streng, Strafrechtliche Sanktionen (Fn. 32), S. 148 f.; Kett-Straub, Lebenslange Freiheitsstrafe (Fn. 3), S. $277 \mathrm{ff}$.

73 Kett-Straub, Lebenslange Freiheitsstrafe (Fn. 3), S. 279 m. w. N.

$74 \mathrm{Zu}$ sehr langen Verbüßungszeiten und Fällen der Entlassung kurz vor dem Tod Dessecker, Dauer und Gründe der Beendigung (Fn. 11), S. 19 f.

75 Lesenswert zur lebenslangen Freiheitsstrafe in England und Wales die allerdings schon vor Inkrafttreten des CJA 2003 erschienene Darstellung von D. Van Zyl Smit, Taking life imprisonment seriously in national and international Law, Den Haag u.a. 2002, S. $78 \mathrm{ff}$.

76 Ein historischer Abriss über die wechselvolle Geschichte der Zuständigkeiten und Voraussetzungen der vorzeitigen Entlassung in England und Wales findet sich bei N. Padfield, England and Wales, in: Padfield et al. (Fn. 22), S. $105 \mathrm{ff}$.

77 R (Anderson) v. Secretary of State for the Home Department vom 25. 11. 2002, [2003] 1 AC 837. 
Das Gericht muss sich bei seiner Entscheidung an den Strafzumessungsrichtlinien (schedule 21 zu Art. 269-277 des CJA 2003) orientieren. Wie oben schon beschrieben, ist der entscheidende Punkt dabei die Schwere der Tat. Sind keine mildernden Umstände ersichtlich, ${ }^{78}$ sind dem Gericht die Hände gebunden und es muss in den beschriebenen Fällen außergewöhnlicher Schwere eine vorzeitige Entlassung ausschließen. Dass eine Abweichung von diesen Vorgaben möglich ist, wird stets betont, sie muss aber ausführlich und in Auseinandersetzung mit den vorgegebenen mildernden Umständen begründet werden. ${ }^{79}$ Schedules sind Ausführungsbestimmungen, die als Bestandteil des Gesetzes gelten. ${ }^{80}$

Bei lebenslangen Freiheitsstrafen, bei denen eine bestimmte Mindestverbüßungsdauer ausgesprochen worden ist, finden spezialpräventive Gesichtspunkte zusätzlich Berücksichtigung, weil auch nach Verbüßung des tariff eine Entlassung nicht automatisch erfolgt. Es bedarf vielmehr der positiven Prognose einer speziellen Kommission (Lifer Panel of the Parole Board), wegen der differenzierten Risikokriterien verbleiben die Gefangenen, wie erwähnt, oft noch viele weitere Jahre nach Verbüßung des tariff in Haft. ${ }^{81}$ Bei den Gefangenen, die eine whole life order erhalten haben, werden spezialpräventive Erwägungen, etwa im Hinblick auf eine positive Entwicklung des Betroffenen, sein Resozialisierungsinteresse oder auch das der Gesellschaft, hingegen nicht mehr angestellt, die Vergeltungsfunktion der Strafe überstrahlt die anderen möglichen Strafzwecke. Dies wurde von englischen Gerichten mehrfach betont und auch mit „dem bewährten Prinzip“ des Common Law begründet, nach dem die Strafe im Verhältnis „zu allen relevanten Umständen der Tat und des Täters, der sie begangen hat" stehen müsse. In Anbetracht, dass englische Richter diese Möglichkeit als ultima ratio nutzen müssten und tatsächlich auch nutzten, seien sie für die schwersten Straftaten als angemessen zu betrachten. ${ }^{82}$

In den letzten Jahren wurde gleichwohl das Problem gesehen, dass eine nicht reduzierbare, bis zum Tod zu vollstreckende Freiheitsstrafe dennoch eine unmenschliche

78 Sie sind ebenfalls in schedule 21 aufgelistet und umfassen in der Parallelwertung des deutschen Strafrechts Faktoren, die die Annahme des Vorsatzes entfallen lassen und nur zu einer Aburteilung wegen Körperverletzung mit Todesfolge führen würden; als Notwehr, Putativnotwehr oder Tötung auf Verlangen zu werten wären oder schuldmindernd geltend gemacht werden könnten. Auch das Alter kann ein solcher Faktor sein.

79 Crown Prosecution Service, Legal Guidance: Mandatory Life Sentences in murder cases (http:// www.cps.gov.uk/legal/s_to_u/, abgerufen am 20.11.2013).

80 Nach Sec. 269 CJA 2003 kann der Lordkanzler (üblicherweise gleichzeitig Justizminister) nach Konsultation mit dem Sentencing Council eine Änderung bewirken. Nach sec. 330 (5) des CJA 2003 müssen dann beide Häuser des Parlaments der Änderung zustimmen.

81 Padfield, England and Wales (Fn. 22), S. 109.

82 Court of Appeal in R. v. Oakes and others [2012] EWCA Crim 2435 m. w. N. 
Strafe nach Art. 3 EMRK darstellen könne. ${ }^{83}$ Entscheidend nach der bislang herrschenden englischen Auffassung ist aber, dass prinzipiell eine Möglichkeit bestehen bleibe, dass der Betroffene dann auf freien Fuß komme, wenn die Fortsetzung nach keinem Strafzweck mehr gerechtfertigt erscheine. Für solche Fälle habe der Innenminister nach sec. 30 des Crime (Sentences) Act 1997 ein Begnadigungsrecht. ${ }^{84}$ Darin ist vorgesehen, dass ein Gefangener dann entlassen werden kann, wenn „außergewöhnliche Gründe existieren, die seine Entlassung aus humanitären Gründen rechtfertigen ". Erscheint diese gesetzliche Formulierung noch relativ offen, hat eine ministerielle Verordnung ${ }^{85}$ das Ermessen faktisch erheblich reduziert - nur Gefangene, die lebensbedrohlich erkrankt sind (wobei die verbleibende Lebenserwartung sogar ein Richtwert von drei Monaten angegeben wird!) oder durch einen Schlaganfall oder vergleichbare Erkrankungen dauerhaft bettlägerig oder gelähmt sind, sollen in Frage kommen.

Für die Niederlande ist oben schon geschildert worden, dass vorwiegend aus Vergeltungsgründen in seltenen Fällen eine lebenslange Freiheitsstrafe verhängt wird, die nicht aussetzbar ist. An eine Wiedereingliederung der solchermaßen Verurteilten ist prinzipiell nicht gedacht, wodurch eine erhebliche Spannung zu dem an sich im niederländischen Recht verankerten Resozialisierungsziel entsteht. Es ist nach bisher herrschender Auffassung auf lebenslange Freiheitsstrafen schlicht nicht anwendbar, sie soll außerhalb der üblichen Grundsätze stehen. ${ }^{86}$ Das macht auch das äußerst strenge Gnadengesetz, das seit 1988 in Kraft ist und die Begnadigung im Falle lebenslanger Freiheitsstrafe regelt, deutlich. ${ }^{87}$ Eine Entlassung ist danach nur möglich, wenn „ein Umstand, dem das Gericht im Moment der Strafzumessung nicht genug Beachtung schenkte oder schenken konnte" vorliegt, bei ausreichender Berücksichtigung dazu geführt hätte, dass das Gericht „eine andere Strafe oder Maßnahme oder Freispruch gewählt hätte. " Diese Variante kommt einer Wiederaufnahme aus Strafzumessungsgründen gleich, ist für die Praxis offenbar aber irrelevant. Der zweite Begnadigungsgrund ist, dass „keinem Strafzweck sinnvollerweise mehr gedient werden kann". Wiederholt wurden Entlassungsanträge abgelehnt, weil die Antragsteller

83 Weitgehend vor allem Richter Laws in R (Wellington) v. Secretary of State for the Home Department [2008] UKHL 72, der zu bedenken gab, dass die Inhaftierung ohne Hoffnung auf Freilassung in verschiedener Hinsicht der Todesstrafe gleichkomme - "in diesem Fall ist der angeblich unveräußerliche Wert des Lebens des Gefangenen allein auf sein Überleben reduziert: auf nichts mehr als zu atmen und, wenngleich zweifellos unter anständigen Umständen, verwahrt zu werden. Dies ist ein bloßes Lippenbekenntnis zum Wert des Lebens, keine Gewährleistung.“.

84 Entsprechend formulierte die britische Regierung in ihrer Einlassung in EGMR, Vinter u.a../. Großbritannien (GK) (Fn. 1), Rn. 94.

85 Prison Service Order 4700 (Indeterminate Sentence Manual), Kapitel 12 (www.justice.gov.uk/offenders/psos/pso-4700-indeterminate-sentence-manual, abgerufen am 20.11.2013).

86 Entsprechend kritisch van Hattum, Levenslang post Vinter (Fn. 19), S. 1964.

87 Art. 2 des niederländischen Gnadengesetzes (Wet van 23 december 1987, houdende regelen voor de indiening en behandeling van en de beschikking op verzoekschriften om gratie, kurz: Gratiewet). 
eben dies nicht darlegen konnten. ${ }^{88}$ Interessant ist, dass bei der Prüfung solcher Anträge offensichtlich in erheblichem Maße auch Opferbelange einfließen. In einem der erfolglosen Anträge war die Ablehnung auch damit begründet, dass die Angehörigen des Mordopfers keinerlei psychologische Fortschritte bei der Bewältigung des durch die Tat erlittenen Traumas gemacht hätten. Für die Entscheidung wurden Stellungnahmen der Angehörigen der Mordopfer eingeholt und sie wurden von Gutachtern untersucht. ${ }^{89}$

Insgesamt werden die bestehenden Regelungen trotz der geringen Zahl von Verurteilungen zu lebenslanger Freiheitsstrafe sowohl von Seiten der Rechtswissenschaft als auch von Lobbygruppen angegriffen. ${ }^{90}$ Sie wurde als „indirekte Version der Todesstrafe “ und als inhuman bezeichnet. ${ }^{91}$ Als Konsequenz wurde - bislang erfolglos ${ }^{92}$ - die Einführung einer justiziellen Überprüfung nach 15 Jahren gefordert, die die fortbestehende Gefährlichkeit des Verurteilten zum Gegenstand haben und weitgehend dem Verfahren für die vorzeitige Entlassung bei zeitigen Freiheitsstrafen entsprechen soll. ${ }^{93}$

Das spanische Recht sieht für die zeitigen Freiheitsstrafen, die es allein kennt, prinzipiell eine Entlassungsmöglichkeit ${ }^{94}$ nach Verbüßung von drei Vierteln der Strafzeit vor. Voraussetzung für eine bedingte Entlassung, gegebenenfalls in Hausarrest, ist eine günstige Sozialprognose, außerdem müssen die Gefangenen innerhalb des gestuften Vollzugs vor der Entlassung eine Zeit in der niedrigsten Sicherheitsstufe (vergleichbar dem offenen Vollzug in Deutschland) untergebracht gewesen sein. Die spanische Praxis wurde mit der Einführung des Codigo Pénal 1995 insofern einer erheblichen Wandlung unterworfen, als zuvor großzügige Anrechnungsregelungen für im Vollzug abgeleitete Arbeit galten (Prinzip der good time), die nunmehr wegfielen. Der Anstieg der spanischen Gefangenenzahlen wird unter anderem darauf zurückgeführt, dass sich die spanische Strafzumessungspraxis an diese Neuerungen nicht anpasste. ${ }^{95}$

88 Rutten, Lifelong Punishment (Fn. 18), S. 11.

89 Allerdings scheinen diese Opfereinschätzungen konkret vor allem in den Fällen lebenslanger Maßregelunterbringung vorzukommen und in Amnestiefragen nur abstrakt einzufließen, vgl. Rutten (Fn. 18), S. 34 ff.

90 Z. B. durch das Forum levenslang. Diese Stiftung hat sich eine Humanisierung der (Vollstreckung der) lebenslangen Freiheitsstrafe zum Ziel gesetzt und diskutiert z. B. auch die Konsequenzen aus Rechtsprechung des EGMR für das niederländische Recht, www.forumlevenslang.nl.

91 G. de Jonge, G., Nederland is nog niet klaar met de doodstraf, Justitiële verkenningen (37) 2011, S. 65 (67).

92 M. Moerings, The Netherlands, in: Padfield et al. (Fn. 22), S. 300 (302).

93 Wetsvoorstel VI voor levenslanggestraften, juli 2011, www.forumlevenslang.nl (abgerufen am 21.11.2013).

94 Allgemeine königliche Amnestien sind mit Inkrafttreten der spanischen Verfassung abgeschafft worden. Ein Gnadenrecht der spanischen Regierung besteht noch, wird aber wenig genutzt.

95 Larrauri/Cid, Spanien (Fn. 9), S. 818 ff. 
Einige der gesetzlichen Verschärfungen der jüngeren Zeit sind Resultate des Kampfes gegen die baskische Separatistenorganisation ETA. ${ }^{96}$ Außerdem war Spanien mit den Madrider Anschlägen im März 2004 auch Ziel islamischen Terrors, die aufsummierten Einzelstrafen für die Täter beliefen sich auf mehrere tausend Jahre. Für alle Täter mit terroristischen Hintergrund ist eine positive Prognose auch davon abhängig, dass sie sich von ihren Zielen distanzieren und bei den Hinterbliebenen bzw. Opfern entschuldigen. ${ }^{97}$ Liegt außerdem die zu verbüßende Freiheitsstrafe niedriger als die Hälfte der nominell verhängten Strafe, was bei terroristischen Straftaten mit mehreren Opfern regelmäßig der Fall ist, so kann sie prinzipiell nicht ausgesetzt werden. Sogar die Schaffung ihrer Voraussetzungen, d. h. das Gewähren von Lockerungen und die Überstellung in den offenen Vollzug, sind vom Tatgericht zwingend auszuschließen. Die zugrundlegende Regelung des Art. 78 Abs. 2 CP ist schon bei seiner Einführung 1995 als zu stark generalpräventiv orientiert kritisiert worden. ${ }^{98}$ In der Tat wirft eine Verbüßungszeit von nunmehr sogar 40 Jahren die Frage auf, wie sie sich mit dem Resozialisierungsgebot des Art. 25 Abs. 2 der spanischen Verfassung verträgt bzw. wie viel Hoffnung sie auf die Rückkehr in ein bürgerliches Leben übrig lässt. ${ }^{99}$ Nicht ganz klar ist außerdem das Verhältnis des Art. $78 \mathrm{CP}$ zum spanischen Strafvollzugsgesetz, das dem Strafvollstreckungsrichter ${ }^{100}$ die Befugnis einräumt, im Verlauf der Vollstreckung bei guter Sozialprognose Gefangene von einem strengeren in ein weniger strenges Vollzugsregime zu verlegen und so den Weg zu einer vorzeitigen Entlassung zu ebnen. Auch dieser Weg ist jedoch ein langer, die Verlegung ist im Fall der Höchststrafe frühestens nach 32 Jahren möglich (4/5 der Strafe), eine bedingte Entlassung frühestens nach 35 Jahren.

96 Cid/Tébar, Spain (Fn. 22), S. 367. Nach der Auflösung der ETA wird allerdings davon ausgegangen, dass sich der Anwendungsbereich der Normen verringert, de la Cuesta, Basque Country (Fn. 26); Cid (oben Fn. 23).

97 Ley Orgánica 1/1979, de 26 de septiembre, General Penitenciaria, Art. 72; Cid/Tébar, Spanien (Fn. 9), S. 371.

98 Cerezo Mir, Spanien (Fn. 49), S. 859.

99 Exemplarisch wurde eingangs der Fall einer baskischen Terroristin geschildert, für die noch die 30Jahre-Grenze bestimmend ist. Außerdem sollten als günstigere rechtliche Regelungen noch die alten Anrechnungsregeln für geleistete Arbeit gelten. Hierbei herrschte eine gewisse Zeit darüber Konsens, dass dieser Abzug von der als selbständig betrachteten „condena“, also der zu verbüßenden Strafe von 30 Jahren, erfolgen sollte. Nach einer Rechtsprechungsänderung des Tribunal Supremo (Obersten Gerichthofs) muss nunmehr der Abzug jedoch von der nominell verhängten Freiheitsstrafe erfolgen, so dass bei Freiheitsstrafen von mehreren hundert Jahren eine vorzeitige Entlassung zumindest auf diesem Wege nicht in Betracht kommt. Die große Kammer des EGMR hat Spanien jedoch dahingehend verurteilt, dass diese Rechtsprechungsänderung gegen das Rückwirkungsverbot aus Art. 7 EMRK verstößt (EGMR [GK] Del Rio Prada./. Spain, Fn. 1). Im Fall der Beschwerdeführerin galt die die nominell verhängte Strafe von 2232 Jahren nach etwa 21 Jahren als verbüßt. Die Beschwerdeführerin ist bereits freigelassen worden, die Schätzungen gehen von mindestens weiteren 50-60 Betroffenen aus (El País vom 23.10. und 28.10.2013). Argumentiert wurde vom EGMR allein mit der Rückwirkungsproblematik; auf das Spannungsverhältnis zum Resozialisierungsgebot der spanischen Verfassung ist nicht eingegangen worden.

100 Ley Orgánica 1/1979, de 26 de septiembre, General Penitenciaria, Art. 76 ff. (Strafvollstreckungsrichter gibt es seit einer größeren Reform im Jahr 1996). 
Die Freiheitsstrafen, die der Strafgerichtshof für das ehemalige Jugoslawien verhängt hat, werden nach Maßgabe völkerrechtlicher Abkommen in verschiedenen europäischen Staaten vollstreckt (Art. 27 S. 1 des Statuts). ${ }^{101}$ Im eingangs skizzierten Fall ist der Verurteilte inzwischen zur Vollstreckung nach Estland überstellt worden; im einzigen weiteren Fall, bei dem auf eine lebenslange Freiheitsstrafe erkannt worden ist, hat Deutschland die Vollstreckung übernommen. ${ }^{102}$ Fraglich ist, nach welchem Recht sich eine vorzeitige Beendigung der Haft richtet. Das Statut ist hierbei unergiebig (und damit im Hinblick auf den Bestimmtheitsgrundsatz hochproblematisch), ${ }^{103}$ weil es in Art. 27 S. 2 des Statuts nur bestimmt, dass die Strafverbüßung sich „nach den geltenden Rechtsvorschriften des betreffenden Staates“ richtet und „der Aufsicht des Internationalen Strafgerichtshofes“ unterliegt. In Art. 28 des Statuts wird weiter geregelt, dass in dem Fall, in dem für den Verurteilten nach den Rechtsvorschriften des Staates, in dem er seine Freiheitsstrafe verbüßt, eine „Begnadigung oder Umwandlung “ der Strafe in Frage kommt, der Gerichtshof informiert werden muss und dessen Präsident sodann über die Entlassung im Einvernehmen mit den Richtern „im Interesse der Rechtspflege und der allgemeinen Rechtsgrundsätze“ entscheidet. Diese Situation wurde allgemein als unbefriedigend empfunden. Mit einer Reihe von Modellabkommen und begünstigt durch die rechtlichen Fortschritte im Zusammenhang mit der Errichtungen des ständigen Internationalen Strafgerichtshofs (IStGH) wurde versucht, einigermaßen einheitliche Maßstäbe zu setzen, die in der Regel auf eine Zwei-Drittel-Verbüßung hinauslaufen. ${ }^{104}$ Wie eine Mindestverbüßungszeit für zu lebenslanger Freiheitsstrafe Verurteilte aussehen soll, ist damit nicht gesagt - geht man von den verhängten hohen zeitigen Freiheitsstrafen (bis 40 Jahre) aus, kann man aber einen Anhaltspunkt von zwei Dritteln dieser Zeit gewinnen.

Für vom IStGH verhängte Strafen ist nach deutschem Recht ${ }^{105}$ anerkannt, dass im Falle einer Vollstreckungsübernahme keine durch ein Exequatur-Verfahren nach IRG gewissermaßen „zu eigen gemachte“ Strafe, sondern eine fremde, nämlich die

101 Die Zusammenarbeit mit Deutschland ist in Bezug auf die Vollstreckung der Urteile in $\ 5$ Abs. 2 Jugoslawien-Strafgerichtshof-Gesetz i. V. m. $\$ 41$ Abs. 2 IStGHG (Gesetz über die Zusammenarbeit mit dem Internationalen Strafgerichtshof) geregelt.

102 http://www.icty.org/x/cases/galic/cis/en/cis_galic_en.pdf und http://www.icty.org/x/cases/milan_lukic_sredoje_lukic/cis/en/cis_lukic_lukic_en.pdf (beide abgerufen am 2.5.2014).

103 Vgl. auch Van Zyl Smit, Strafzumessung (Fn. 30), S. 942.

104 Zuletzt z. B. Prosecutor v Krajišnik (President's Decision) IT-00-39-ES, 08.11.12, Rn. 24; zu dieser "über den Daumen gepeilten" und dementsprechend rechtsstaatlich problematischen Regelung ausführlich R. Mulgrew, Releasing International Prisoners, in: M. Herzog-Evans (Hrsg.), Offender Release and Supervision: The Role of Courts and the Use of Discretion, Nijmegen (im Erscheinen).

105 Dieser Entwicklung ging ein mühsamer Lernprozess durch die Erfahrungen in einem der Fälle, in denen Deutschland die Vollstreckung einer Strafe des Jugoslawien-Gerichtshofs übernommen hatte, voran, vgl. P. Wilkitzki, Erläuterungen zum Gesetz über die Zusammenarbeit mit dem Internationalen Strafgerichtshof für das ehemalige Jugoslawien (Jugoslawien-Strafgerichtshof-Gesetz), BadenBaden 2008, $\int 5$ Rn. 5 ff. 
des Gerichtshofs, vollstreckt wird. Im Einzelnen bedeutet dies, dass die Vollstreckung der Freiheitsstrafe in der vom Gerichtshof mitgeteilten Höhe ( $\mathbb{} 41$ Abs. 2 S. 1 Gesetz über die Zusammenarbeit mit dem Internationalen Strafgerichtshof, IStGHG) erfolgt. Nach den Schwierigkeiten bei den ersten beiden Vollstreckungsübernahmen auch für diese Vollstreckungsübernahmen eine eigene gesetzliche Grundlage geschaffen $(\mathbb{5}$ Abs. 2 Jugoslawien-Strafgerichtshof-Gesetz i. V. m. $\$ 41$ Abs. 2 IStGHG). Vollstreckbar sind damit lebenslange Freiheitsstrafen und zeitige Freiheitsstrafen bis zu einer Höchstdauer von 30 Jahren. Die $\mathbb{S} \$ 57$ und 57a StGB gelten nicht, sondern die Beendigung der Vollstreckung kann (nur) auf Grund einer Anweisung des Gerichtshofes erfolgen. Ferner bleibt seine umfassende Zuständigkeit für alle mit der Vollstreckung der Strafe zusammenhängenden Entscheidungen bestehen (damit sind Vollzugslockerungen, die ein Verlassen der Anstalt beinhalten, gemeint). Das deutsche Strafvollzugsrecht ist nur subsidiär und ohne sein Beschwerde- und Gerichtsverfahren anwendbar.

Damit fehlt es an einer klaren Rechtsgrundlage für eine bedingte Entlassung der beiden bislang zu lebenslanger Freiheitsstrafen verurteilten Täter. Der nach Deutschland überstellte Betroffene verbüßt seine Strafe (unter Anrechnung der Untersuchungshaft) seit gut 14 Jahren - nach deutschem Recht müsste sich die Strafvollstreckungsbehörde nun langsam Gedanken machen, wie sie die Entscheidung der Vollstreckungskammer zur etwaigen Entlassung vorbereitet, für diesen Gefangenen hingegen werden die entsprechenden Anstrengungen wohl unterbleiben. Angesichts der - in der Parallelwertung des deutschen Rechts - anzunehmenden „Schwere der Schuld“ mag dies hinnehmbar sein. Dennoch ist eine gewisse Reibung mit der Verfassungsrechtsprechung zur Berücksichtigung des Resozialisierungsgrundsatzes bei lebenslangen Freiheitsstrafen ${ }^{106}$ bei dieser immerhin auf deutschem Boden vollstreckten Strafe nicht zu leugnen.

\section{Europäisches Recht: Die EMRK und die Rechtsprechung des EGMR}

\section{Die Grundlagen der EMRK}

Art. 3 EMRK bestimmt, dass niemand der Folter oder unmenschlicher oder erniedrigender Behandlung oder Strafe unterworfen werden darf. In Art. 5 Abs. 1 EMRK ist das Recht auf Freiheit und Sicherheit niedergelegt. Die Freiheit darf nur auf die gesetzlich vorgeschriebene Weise entzogen werden und nur in vom Art. 5 Abs. 1 S. 2 EMRK aufgezählten Fällen - einschlägig ist bei Freiheitsstrafen lit. a), „nach Verurteilung durch ein zuständiges Gericht“. Nach Art. 5 Abs. 4 EMRK hat außerdem jede Person, die festgenommen oder der die Freiheit entzogen ist, das Recht zu beantragen, dass ein Gericht über die Rechtmäßigkeit der Freiheitsentziehung entscheidet und ihre Entlassung anordnet, wenn sie nicht rechtmäßig ist. 
Der Schutzbereich von Art. 3 EMRK lässt sich anhand der umfangreichen Rechtsprechung des EGMR so umreißen, dass die physische und psychische Integrität der Grundrechtsträger geschützt sind, und eine Verletzung sich in der „Missachtung der Person in ihrem Menschsein " manifestiert. ${ }^{107}$ Außerdem wird davon ausgegangen, dass alle internationalen Menschenrechtsinstrumente (d. h. insbesondere die Allgemeine Erklärung der Menschenrechte, AEMR, und der Internationale Pakt für bürgerliche und politische Rechte, IPBPR) mit den korrespondierenden Regelungen unantastbare Grundwerte demokratischer Gesellschaften schützen und in der Achtung der Menschenwürde wurzeln. ${ }^{108}$ Allerdings fehlt es in der EMRK an einer expliziten Bezugnahme auf die Menschenwürde, anders als etwa in Art. 1 AEMR oder auch Art. 10 IPBPR. Dort heißt es ausdrücklich zur Situation von Inhaftierten: „Jeder, dem seine Freiheit entzogen ist, muss menschlich und mit Achtung vor der dem Menschen innewohnenden Würde behandelt werden.“

In Bezug auf die Tatbestandsvarianten Folter, unmenschlicher Behandlung oder Strafe sowie erniedrigender Behandlung oder Strafe wird von einem Stufenverhältnis ausgegangen, wobei die Folter die gravierendste Erscheinungsform darstellt und sowohl in der Ausarbeitung der Konvention als auch in vielen Einzelfällen Bezugspunkt der Diskussion war. Am anderen Ende des Kontinuums steht die erniedrigende Behandlung. ${ }^{109}$ Anders als der Gerichtshof war die Europäische Menschenrechtskommission in einem horizontal angelegten Modell offenbar von einem Oberbegriff der unmenschlichen Behandlung ausgegangen, der auf der einen Seite Folter als absichtsvolle, ungerechtfertigte Zufügung von Leiden einschloss und auf der anderen Seite als erniedrigende Behandlung definierte, was ein Individuum „gröblich vor anderen demütigt oder was ihn dazu treibt, gegen sein Gewissen zu handeln“ “. ${ }^{110} I n$ den Entscheidungen des EGMR ist nicht immer klar, warum welche Tatbestandsvariante als Anknüpfungspunkt gewählt wird. ${ }^{111}$ Parallelen werden zum Schutzzweck des Art. 1 GG insofern deutlich, als es in beiden Vorschriften um die Wahrung der Fremd- und Selbstachtung geht, d. h. auch eine subjektive Komponente Berücksichtigung findet.

107 C. Grabenwarter/K. Pabel, Europäische Menschenrechtskonvention, 5. Auflage, München u.a. 2012, S. 163.

108 R. Esser, in: V. Erb/R. Esser/U. Franke/K. Graalmann-Scheerer/H. Hilger/A. Ignor (Hrsg.), Löwe/ Rosenberg: Die Strafprozeßordnung und das Gerichtsverfassungsgesetz, Bd. 11, Berlin u.a. 2012, Art. 3 EMRK/Art. 7 IPBPR Rn. 1; J. Meyer-Ladewig Menschenwürde und Europäische Menschenrechtskonvention NJW 2004, S. 981 (982).

109 M. D. Evans, Getting to Grips with Torture, International and Comparative Law Quarterly (51) 2002, S. 365 (370); R. Bank, Das Verbot von Folter, unmenschlicher oder erniedrigender Behandlung oder Strafe, in: R. Grote/ T.Marauhn (Hrsg.), EMRK/GG. Konkordanzkommentar zum europäischen und deutschen Grundrechtsschutz, Tübingen 2002, Rn. 6.

110 "...grossly humiliates him before others or drives him to act against his conscience" (meine Übersetzung); "Greek Case”, European Commission of Human Rights, 5. 11. 1969, Rn. 186. Das ursprüngliche System der EMRK sah die Europäische Kommission für Menschenrechte als dem Gerichtshof vorgelagerte Beschwerdeinstanz vor. Sie wurde mit dem 11. ZP 1998 abgeschafft.

111 Evans, Torture (Fn. 109), S. 373; Bank, Verbot der Folter (Fn. 109), Rn. 39 ff. 
In jüngerer Zeit wird in Zusammenschau insbesondere mit Art. 8 EMRK und gerade auch im Hinblick auf Fragen des Strafvollzugs für Art. 3 EMRK eine Entwicklung in Richtung eines umfassenden Schutzes der Menschenwürde deutlicher. ${ }^{112}$

Ein weiteres Problem ist, dass Art. 3 EMRK prinzipiell schrankenlos gilt, gleichzeitig aber (wie für Art. 1 GG) anerkannt ist, dass nur solche Eingriffe die Integrität des Einzelnen verletzen, die eine bestimmte Schwelle überschreiten. ${ }^{113}$ Neben diese quantitative Relativierung, die Bagatellbeeinträchtigungen aus dem Schutzbereich herausnehmen sollen, tritt eine qualitative: So wird argumentiert, dass sich die Freiheitsstrafe aus dem Anwendungsbereich prinzipiell „herausdefinieren lasse“, denn sie stelle einerseits stets einen erheblichen Eingriff in die körperlich und psychische Integrität dar, sei aber andererseits schon nach dem Wortlaut („Behandlung oder Strafe“) immer dann legitim, wenn sie nicht unmenschlich oder erniedrigend sei. ${ }^{114}$ Damit wird im Bereich der Strafen und der Strafzumessung eine Verhältnismäßigkeitsprüfung eröffnet, die letztlich den Schutzbereich erst bestimmen kann. Sie lässt den Gesetzgebern der Mitgliedstaaten viel Spielraum und sorgt für die reiche Kasuistik. Anerkannt ist für den Vollzug der Strafe, dass eine Kumulation von schlechten Haftbedingungen (vor allem Überfüllung und mangelhafte hygienische Bedingungen) zu einer Verletzung von Art. 3 EMRK führen kann, auch wenn die unmenschliche Behandlung nicht intentional erfolgt. ${ }^{115}$ Für die Verhängung der Strafe wird angenommen, dass eine grob unverhältnismäßige (grossly disproportionate) Strafe zu einer Verletzung von Art. 3 EMRK führen kann. Bislang aber gibt es keinen Fall, in dem Gerichtshof davon ausgegangen ist, dass sich die Strafzumessung nicht mehr in dem den Mitgliedstaaten zugestandenen Spielraum bewegt hätte. Er begründete dies stets damit, dass die Erheblichkeitsschwelle für Art. 3 EMRK nicht erreicht gewesen sei. $^{116}$

Die prozessualen Garantien des Art. 5 Abs. 4 EMRK, d. h. die richterliche Haftüberprüfung, gelten prinzipiell für alle Fälle der Freiheitsentziehung, die in Art. 5 Abs. 1 EMRK aufgeführt sind. Die Kontrollfunktion soll aber schon dadurch erfüllt sein, dass ein Gericht eine Person zu einer bestimmten Freiheitsstrafe verurteilt hat. Die Notwendigkeit weitergehender Überprüfungen während des Vollzugs dieser Frei-

112 Evans, Torture (Fn. 109), S. 373; J. Meyer-Ladewig (Fn. 108), S. 981 (983); Bank, Verbot von Folter (Fn. 109), Rn. 9; EGMR vom 29.4.2002, Pretty./.United Kingdom, Nr. 2346/02 (NJW 2002, S. 2851) Rn. 52.

113 Grabenwarter/Pabel, EMRK, (Fn. 107), S. 163; Evans, Torture (Fn. 109), S. 373.

114 Vgl. z. B. EGMR vom 12.2.2008, Kafkaris./. Zypern, Nr. 21906/04; Bank (Fn. 109), Rn. 13 m.w.N.

115 Evans, Torture (Fn. 109), S. 372 m.w.N., Leitentscheidungen sind EGMR vom 15.7.2002. Kalashnikov./.Russland, Nr. $47095 / 99$ und vom 19.4.2001, Peers./.Griechenland, Nr. 28524/95.

116 J. Meyer-Ladewig, EMRK: Europäische Menschenrechtskonvention, 3. Aufl., Baden-Baden 2011, Art. 3 Rn. 57 m.w.N. 
heitsstrafe soll hingegen nicht aus Art. 5 Abs. 4 EMRK abzuleiten sein. ${ }^{117}$ Diese enge Lesart ist durch die historischen Wurzeln im habeas corpus-Gedanken bedingt, nach denen primär in der Untersuchungshaft die Rechtmäßigkeit der Freiheitsentziehung sowohl zu Beginn als auch später in regelmäßigen Zeitabständen überprüft werden können muss, weil sich im Laufe der Zeit neue Fragen ergeben können. ${ }^{118}$ Der Grundgedanke ist aber später durchaus auch auf Fälle von Freiheitsentziehung, die präventiven Charakter haben, d. h. etwa die Unterbringung psychisch Kranker bzw. Sicherungsverwahrter, übertragen worden. ${ }^{119}$

\section{Die Entwicklung der EGMR-Rechtsprechung zu nicht aussetzbaren lebenslangen Freiheitsstrafen}

Die Weichen für die jetzige Haltung des EGMR zur nicht aussetzbaren lebenslangen Freiheitsstrafe wurden im Erwachsenenbereich ${ }^{120}$ im Jahr 2008 mit der Entscheidung Kafkaris/Zypern gestellt. ${ }^{121}$ Der Betroffene war zu einer lebenslangen Freiheitsstrafe verurteilt worden, während seiner Inhaftierung wurde die Entlassungspraxis geändert, so dass er nunmehr auf eine Gnadenentscheidung des zyprischen Präsidenten angewiesen war. Er argumentierte u. a. diese Ungewissheit habe starkes physisches und psychisches Leid bei ihm verursacht und es sei ihm alle Hoffnung genommen, jemals entlassen zu werden. Auch wenn im konkreten Fall die Beschwerde nicht erfolgreich war (die Entscheidung der Großen Kammer erging relativ knapp mit 10:7 Stimmen), wurden wichtige Kriterien entwickelt, die die weitere Rechtsprechung des Gerichtshofs prägten. In früheren Entscheidungen war schon der Begriff der „nicht reduzierbaren Freiheitsstrafe" (irreducible life sentence) eingeführt worden, sie könne unter Umständen im Hinblick auf Art. 3 EMRK problematisch sein. ${ }^{122}$ Dieser Begriff wurde im komplizierten und politisch brisanten ${ }^{123}$ Kafkaris-Fall dahingehend konkretisiert, dass dies dann der Fall sei, wenn „de jure und de facto “ eine Entlassung ausgeschlossen sei. ${ }^{124}$ Letztlich wurde wegen der Begnadigungsmöglichkeit angenommen, dass eine solche Hoffnung auf Entlassung, wenngleich vage, gegeben sei und deshalb kein Verstoß gegen Art. 3 EMRK vorliege. Ein ausführliches Minder-

117 O. Dörr, Freiheit der Person, in: Grote/Marauhn (Hrsg.): EMRK/GG (Fn. 109); EGMR vom 18.6.1971,, De Wilde u.a./Belgien, 2832/66; EGMR vom 17.1.2012 (4. Kammer), Vinter u.a../.Vereinigtes Königreich, Nr. 66069/09, 130/10 and 3896, Rn. 103.

118 Meyer-Ladewig, EMRK (Fn. 116), Art. 5 Rn. 84.

119 EGMR v.9.5.2007, 12788/04, NJW 2008, 2320 (Homann./.Deutschland) und EGMR vom28.5.2002 (GK), Stafford./.Vereinigtes Königreich Nr. 46295/99.

120 Für Jugendliche sind lebenslange Freiheitsstrafen ohne Aussicht auf Entlassung schon in früheren Entscheidungen als unvereinbar mit der EMRK angesehen worden, vgl. D. van Zyl Smit (Fn. 5), S. 39 (39) m.w.N.

121 EGMR vom 12.2.2008 (GK), Kafkaris./.Zypern, Nr. 21906/04.

122 EGMR vom 16.10.2001, Einhorn./.Frankreich, Nr. 71555/01, Rn. 27.

123 Das geht aus dem Minderheitsvotum des Richters Borrego Borrego im Kafkaris-Urteil (Fn. 121) hervor.

124 Kafkaris./. Zypern (Fn. 121), Rn. 98. 
heitsvotum distanzierte sich von diesem Schluss, weil die Gnadenpraxis völlig unvorhersehbar sei - damit wurde zum einen materiell das Problem quälender Ungewissheit als Aspekt von Art. 3 EMRK ins Spiel gebracht, zum anderen prozessual das Problem fehlender Schutzmechanismen angesprochen. ${ }^{125}$ In einem weiteren Sondervotum wurde moniert, dass der Gerichtshof die Chance habe verstreichen lassen, endgültig zur Verhängung einer nicht reduzierbaren Freiheitsstrafe als Verstoß gegen Art. 3 EMRK Stellung zu nehmen. ${ }^{126}$

Vier Jahre später ergab sich diese Chance erneut: Im Januar 2012 war darüber zu befinden, ob die gegen die drei englischen Beschwerdeführer als whole life orders verhängten lebenslangen Freiheitsstrafen gegen Art. 3 EMRK verstießen. ${ }^{127}$ Sowohl die Beschwerdeführer als auch drei der sieben beteiligten Richter argumentierten auf der Basis des Kafkaris-Urteils, dass eine konventionskonforme Anwendung der lebenslangen Freiheitsstrafe beinhalten müsse, dass die Betroffenen „Hoffnung für die Zukunft haben können, unabhängig davon, wie schwach sie auch sein mag“. Die (oben unter II 3 geschilderten) rechtlichen Grundlagen bzw. die äußerst strenge Begnadigungspraxis in England verstießen gegen diese Anforderungen. ${ }^{128}$ Auch in diesem Kammerurteil finden sich neue, wichtige Akzente: So wurde von allen Richtern die Annahme getragen, dass irgendwann im Verlauf einer lebenslangen Freiheitsstrafe ein Punkt erreicht sein könnte, an dem die Vollstreckung nicht mehr von Strafzwecken gerechtfertigt sein könnte („can no longer be justified on any legitimate penological grounds [such as punishment, deterrence, public protection or rehabilitation]“). ${ }^{129}$ Akzeptiert wurde also prinzipiell ein dynamisches Moment. Obwohl der Gerichtshof die äußerst restriktive englische Gnadenpraxis und die Abschaffung der Haftprüfung nach 25 Jahren bemängelte, konnte die Mehrheit dennoch im Ergebnis keinen Verstoß gegen Art. 3 EMRK feststellen. Entscheidend war letztlich genau das beschriebene dynamische Moment: So lange noch legitime Strafzwecke, angesichts der Schwere der Verbrechen hier Vergeltung und Abschreckung (,punishment and deterrence“), mit der Inhaftierung erfüllt würden, könne Art. 3 EMRK noch nicht verletzt sein. ${ }^{130}$

\section{Die Entscheidung der Großen Kammer des EGMR im Fall Vinter u.a.}

Die Beschwerdeführer beantragten die Verweisung der Sache an die Große Kammer, die sie wegen deren überragender Bedeutung gem. Art. 43 Abs. 2 EMRK annahm.

125 Teilweise abweichendes Votum der Richterinnen und Richter Tulkens, Cabral Barreto, Fura-Sandström, Spielmann und Jebens zum Kafkaris-Urteil (Fn. 121).

126 Sondervotum des Richters Bratza zum Kafkaris-Urteil (Fn. 121).

127 EGMR (4. Kammer) Vinter u.a./. Großbritannien (Fn. 117).

128 Teilweise abweichendes Votum der Richter Garlicki, David Thór Björgvinsson and Nicolaou in EGMR (4. Kammer) Vinter u.a./. Großbritannien (Fn. 117).

129 EGMR (4. Kammer) Vinter u.a./. Großbritannien (Fn. 117), Rn. 91.

130 EGMR (4. Kammer) Vinter u.a./. Großbritannien (Fn. 117), Rn. 95. 
Der großen Kammer dürfen nach Art. 26 Abs. 5 EMRK nicht die mit der Kammerentscheidung befassten Richter angehören. Eine Ausnahme gilt nur für den jeweiligen national zuständigen Richter, allerdings war im konkreten Fall wegen Ablauf der Amtszeit des britischen Richters tatsächlich auch dieser ausgewechselt. Die Entscheidung der Großen Kammer, die nunmehr einen Verstoß gegen Art. 3 EMRK annahm, erging mit einer überraschend deutlichen Mehrheit von 16:1.

Kompatibel mit Art. 3 EMRK sind danach prinzipiell nur solche lebenslangen Freiheitsstrafen, die eine Aussicht auf Entlassung und eine Möglichkeit der Überprüfung beinhalten. ${ }^{131}$ Die Große Kammer begründet dies mit vier Argumentationslinien: Sie wiederholt die Überlegung zu Dynamik, also, dass sich die Strafzweckgewichtung während der Strafvollstreckung verschieben könne, und folgert praktisch, dass hierüber nur Klarheit gewonnen werden kann, wenn tatsächlich eine Überprüfung erfolgt. Das zweite Argument hat kriminologische und rechtstheoretische Untertöne: Ohne Überprüfung und ohne irgendeine Aussicht auf Entlassung habe der Betroffene letztlich keine Chance, seine Tat tatsächlich „abzubüßen“ („atone for“) - im modernen Strafvollzugsrecht würde man wohl sagen, er habe keine Chance und auch keine Veranlassung, seine Tat zu bearbeiten - da völlig unabhängig von seinen Bemühungen und der Entwicklung seiner Persönlichkeit die Strafe unveränderlich bleibe. Das dritte Argument bezieht sich ausdrücklich auf die Entscheidungen des Bundesverfassungsgerichts. Danach ist eine Freiheitsentziehung ohne eine Chance auf Entlassung mit dem Gedanken der Menschenwürde, wie sie auch in der Konvention zum Ausdruck komme, nicht zu vereinbaren - unabhängig von der Schwere des Delikts. Schließlich beschreibt der Gerichtshof eine Entwicklung, die sich grob als europäisches Bekenntnis zum Resozialisierungsgedanken zusammenfassen lässt. Es handelt sich streng genommen nicht um aus der Konvention entwickelten Gedanken, es wird vielmehr anerkannt, dass sowohl die meisten nationalen Rechtsordnungen in Europa, Instrumente des Europarats und das Europäische Anti-Folter-Komitee neben der Vergeltung zunehmend das Resozialisierungsanliegen des Strafvollzugs betonten. Wichtig ist die Feststellung, dass Gefangene, die eine lebenslange Freiheitsstrafe erhalten haben, diesbezüglich nicht anders behandelt werden dürfen. Abgerundet werden die vergleichenden Überlegungen mit dem Hinweis auf die Praxis der internationalen Strafgerichtshöfe bzw. dem Resozialisierungsgebot in Art. 10 Abs. 3 IPBPR.

Als Konsequenz des Gesagten und in Abkehr von der Kammerentscheidung stellt die Große Kammer fest, dass eine nicht reduzierbare lebenslange Freiheitsstrafe schon zum Zeitpunkt ihrer Verhängung konventionswidrig ist, weil es „unberechenbar wäre, vom Gefangenen zu erwarten, dass er an seiner Resozialisierung arbeitet, ohne zu wissen, ob es zu einem unbestimmten zukünftigen Zeitpunkt einen Mechanismus 
geben wird, der es aufgrund dieser Resozialisierung ermöglichen würde, dass seine Entlassung erwogen wird. " Letztlich wird dies auch unter Rückgriff auf einen allgemeinen Bestimmtheitsgrundsatz begründet. ${ }^{132}$

\section{Würdigung: Das Recht auf Hoffnung als europäisches Menschenrecht?}

\section{Die Herleitung aus Art. 3 EMRK}

Mit der Entscheidung der Großen Kammer in Vinter $u$. a. steht nun fest: Lebenslange Freiheitsstrafen ohne reguläre Entlassungsmöglichkeit sind von vornherein nicht mit der EMRK zu vereinbaren. Die Herleitung dieses Schlusses ist kritisiert worden, was auf den ersten Blick auch berechtigt erscheint: Im abweichenden Votum wird moniert, dass sich das Urteil auf „Standards und Erfordernisse“ („standards and requirements") von Art. 3 bezieht, ohne diese zu erklären und dass pauschal auf die Vorschrift Bezug genommen werde, ohne die Tatbestandsvariante bzw. die in früheren Urteilen entwickelten unterschiedlichen Eingriffsschwellen zu thematisieren. ${ }^{133}$ In der Tat ist die Begründung der Entscheidung kein leuchtendes Beispiel juristischer Argumentationskultur. ${ }^{134}$ Andererseits ist es durchaus möglich, sich die nötigen Prinzipien zur Konkretisierung des Art. 3 EMRK und ihre Anwendung auf den Fall selbst aus dem Urteil zusammenzusuchen; sie stehen auch im Einklang mit den früher aufgestellten und oben teilweise beschriebenen Grundsätzen.

Was also sind die hier angewandten Standards und Erfordernisse des Art. 3 EMRK? Wodurch genau ist der Schwellenwert zur erniedrigenden Behandlung erreicht worden? Die Große Kammer argumentiert unter ausdrücklicher Berufung auf das Bundesverfassungsgericht mit einem unzulässigen Eingriff in die Menschenwürde. Nun ist die Ausfüllung dieses Begriffs bekanntlich hochumstritten, ${ }^{135}$ sie wird vom EGMR auch gar nicht versucht. Seine Operationalisierung des Begriffs lässt sich wohl am besten mit einer „Deutung von Verletzungsvorgang her"136 umschreiben. Diese passt dann auch wieder zu den vom EGMR bislang entwickelten Kriterien, ab wann die Eingangsschwelle zur erniedrigenden - menschenunwürdigen - Behandlung erreicht

132 EGMR, Vinter u.a../. Großbritannien (GK), (Fn. 1), Rn. 122: „...capricious to expect the prisoner to work towards his own rehabilitation without knowing whether, at an unspecified, future date a mechanism might be introduced which would allow him, on the basis of that rehabilitation, to be considered for release.".

133 Teilweise abweichendes Votum des Richters Villiger in EGMR, Vinter u.a../. Großbritannien (GK), (Fn. 1) mit Bezug auf Rn. 121 und 122.

134 Schon früher ist die „miserable Qualität“ von Urteilen des EGMR kritisiert worden, vgl. T. Hörnle, Der Streit um die Sicherungsverwahrung. Anmerkung zum Urteil des 2. Senats des BVerfG vom 4.5.2011 - NStZ 2011, 450, NStZ 2011, S. 488 (490); sie macht dafür (auch) die common-lawTradition der Urteilsfindung verantwortlich.

135 Umfangreiche Auseinandersetzungen finden sich z. B. bei C. Enders, Die Menschenwürde in der Verfassungsordnung: zur Dogmatik des Art. 1 GG, Tübingen 1997 und P. Tiedemann, Menschenwürde als Rechtsbegriff. Eine philosophische Klärung, 2. Aufl., Berlin 2010.

136 Tiedemann, Menschenwürde (Fn. 135), S. 81. Eine solche „Kasuistik klarer Verletzungstatbestände“ zur Identifikation der Garantien des Art. 1 GG empfiehlt P. Badura, Generalprävention und Würde des Menschen, JZ 1964, S. 337 (341). 
ist. Zu fragen ist also, wodurch bei einer nicht reduzierbaren lebenslangen Freiheitsstrafe die psychische oder auch physische Integrität verletzt werden kann bzw. wodurch Leiden verursacht wird. Die Beschwerdeführer argumentierten (und legten entsprechende Gutachten vor), dass die lange Haftzeit bereits persönlichkeitsschädigende Wirkung gehabt habe. Die Tatsache, dass es nicht einmal eine vage Hoffnung auf Entlassung gebe, habe zu einem Zustand der Verzweiflung geführt. ${ }^{137}$

Unterschwellig oder ausdrücklich wird also stets damit argumentiert, dass zu einem menschenwürdigen Dasein eine Entwicklungsmöglichkeit, das Streben nach einem Ziel - kurz: Hoffnung - gehört. Dass dies auf Dauer in Unfreiheit gelingen könnte (die Sinnhaftigkeit des Lebens also etwa dadurch erreicht werden könnte, ein besonders guter Gefangener zu sein), wird von niemandem ernsthaft behauptet. Diese psychologisch und strafvollzugswissenschaftlich lange abgesicherte ${ }^{138}$ Erkenntnis wird in vielen der vom EGMR herangezogenen Texte in verschiedenen Varianten angeführt: Neben dem auch hier bereits zitierten englischen Richter Laws und dem Bundesverfassungsgericht wird z. B. das Europäische Anti-Folter-Komitee genannt, dass davon ausgeht, „dass es unmenschlich ist, jemanden sein Leben lang zu inhaftieren ohne dass er eine realistische Hoffnung hat, entlassen zu werden. "139 Die Fähigkeit des Menschen zur Hoffnung als Element der Menschenwürde - und im Umkehrschluss: die Entwürdigung, wenn man sie ihm nimmt ${ }^{140}$ - gibt letztlich auch den Ausschlag für die Entscheidung der Großen Kammer. Eines der Sondervoten sah sich veranlasst, dies noch deutlicher herauszuarbeiten. Darin heißt es: „Art. 3 umfasst das, was man als‘das Recht zu hoffen` beschreiben könnte. Mehr bedarf es nicht. Das Urteil erkennt implizit an, dass die Fähigkeit zur Hoffnung ein wichtiger und konstituierender Aspekt der menschlichen Persönlichkeit ist. Auch diejenigen, die die abscheulichsten und ungeheuerlichsten Taten begehen und unermessliches Leid über andere bringen, behalten ihre fundamentale Menschlichkeit und tragen in sich die Fähigkeit zum Wandel. ... Ihnen die Erfahrung zu hoffen zu nehmen, hieße ihnen

137 EGMR (GK), Vinter u.a../. Großbritannien, (Fn. 1), Rn. 101; EGMR (GK), Kafkaris./. Zypern (Fn. 121), Rn. 84.

138 Der Begründer der Individualpsychologie Alfred Adler identifiziert das Streben nach einem Ziel als bestimmend für das menschliche Seelenleben bzw. seine Individualität (z. B. in A. Adler, Menschenkenntnis [1927], Studienausgabe, hrsg. von Jörg Ruedi, Göttingen 2007, S. 37). Das Bundesverfassungsgericht zitiert das Gutachten von Liepmann für den 31. Deutschen Juristentag aus dem Jahre 1912, der die Gefangenen als „Ruinen“ beschreibt, wenn sie nach sehr langer Haft nicht länger den Gedanken an eine Vorläufigkeit ihrer Haftsituation bzw. an eine Begnadigung aufrechterhielten (BVerfGE 45, 187, 231).

139 EGMR (GK), Vinter u.a../. Großbritannien, (Fn. 1), Rn. 64; die Aussage stammt aus dem Bericht über den Besuch in der Schweiz im Jahr 2011 (CPT, 25 October 2012 CPT/Inf (2012) 26; abrufbar über www.cpt.coe.int).

140 Oder auch: „The cruelty of a life without hope“, van D. Zyl Smit, The Cruelty of Life without Hope, in: J. Claessen/D. de Vocht (Hrsg.), Humaan strafwerk. Liber Amicorum Gerard de Jonge, Nijmegen 2012, S. 409 Er nimmt mit dem Titel auf die Formulierung im 8. Zusatzartikel zur Verfassung der Vereinigten Staaten, der Entsprechung von Art. 3 EMRK, Bezug. 
einen fundamentalen Aspekt ihrer Menschlichkeit zu nehmen, es wäre erniedrigend (Übers. der Verf.) “141

Bei genauerer Betrachtung wird auch ein utilitaristischer Aspekt deutlich, etwa im ebenfalls zitierten Kommentar zur Empfehlung (2003) 22 zur bedingten Entlassung des Europarats: Danach „,bereitet die Inhaftierung von Personen, die keine Hoffnung auf Entlassung haben, ernste Probleme wenn es darum geht, Anreize zur Kooperation zu schaffen und auf störendes Verhalten zu reagieren, persönlichkeitsentwickelnde Programme bereitzustellen, Vollzugspläne zu erstellen und in Bezug auf die Sicherheit." Diese aus der Strafvollzugswissenschaft vertraute Überlegung hat dem Argument möglicherweise noch mehr Durchschlagskraft verliehen und verknüpft den Aspekt der Menschenwürde mit dem der Resozialisierung. ${ }^{142}$

\section{Entlassungsaussicht de jure und de facto: spezialpräventive Kriterien}

Zur Begründung der Notwendigkeit einer Haftprüfung ist die Strafzweckdiskussion nochmals aufzunehmen. Einerseits streitet der absolute Anspruch auf menschenwürdige Behandlung erfolgreich gegen das Argument, Vergeltung und Abschreckung als Strafzwecke könnten bis zum Tode des Gefangenen seine Inhaftierung legimitieren und eine erneute Überprüfung unnötig machen. Betont wird im Urteil vielmehr das dynamische Moment, das eine Verschiebung der im konkreten Fall zu berücksichtigen Strafzwecke beinhaltet. ${ }^{143}$ Akzeptiert wird vom EGMR, dass spezialpräventive Gründe eine Inhaftierung bis zum Tode im Einzelfall rechtfertigen können, das war im Übrigen auch von den Beschwerdeführern zugestanden worden: Dies ist im Licht von Art. 3 EMRK immer dann zu rechtfertigen, wenn der Betroffene weiterhin ein Risiko für die Gesellschaft darstellt. Der EGMR leitet dies aus einem Drittbezug, nämlich der Verpflichtung des Staates, die Öffentlichkeit zu schützen, ab. Ausdrücklich wird die negative Spezialprävention als Vollzugszweck bezeichnet. ${ }^{144}$

Auch der EGMR verknüpft die Frage nach der Menschenwürde aber mit dem Strafzweck der Resozialisierung. Wieder ist zu bemängeln, dass breite Ausführungen zur Frage zu finden sind, deren Verbindlichkeit unklar ist. Aufhorchen lässt jedenfalls, dass der Gerichtshof sich auf die Lebenslang-Entscheidung des Bundesverfassungsgerichts insofern beruft, als dort die Strafvollzugsbehörden dazu angehalten worden seien, dass sie auch bei zu lebenslanger Freiheitsstrafe Verurteilten auf seine Resozialisierung hinarbeiten müssten und dass diese Resozialisierung „in jeder Gesellschaft verlangt werde, die die Menschenwürde als zentrales Element festgeschrieben habe“. Weiter heißt es „Die gleichen Überlegungen müssen unter dem System der

141 EGMR (GK), Vinter u.a../. Großbritannien, (Fn. 1), Sondervotum der Richterin Power-Forde.

142 EGMR (GK), Vinter u.a../. Großbritannien, (Fn. 1), Rn. 62.

143 EGMR (GK), Vinter u.a../. Großbritannien (Fn. 1), Rn. $111 \mathrm{f}$.

144 EGMR (GK), Vinter u.a../. Großbritannien (Fn. 1), Rn. 108, siehe auch Rn. 99 und Rn. 131. 
Konvention gelten, deren Wesen, wie der Gerichtshof oft betont hat, gerade von der Achtung der Menschenwürde bestimmt wird.“

Für die praktische Umsetzung des Urteils wird vor allem von Bedeutung sein, wann ein nationales System de jure und de facto einen ausreichenden Grund für Hoffnung bzw. eine Entlassungsaussicht bietet. Dass hier entscheidende Probleme lauern, zeigt nicht zuletzt die erwähnte 10:7-Entscheidung in der Entscheidung Kafkaris von 2008 und das angefügte Minderheitsvotum: Für die fünf unterzeichnenden Richterinnen und Richter war schlicht nicht nachzuvollziehen, wie die Mehrheit anhand der ausgebreiteten Argumente dazu kommen konnte, dass das völlig intransparente Gnadenverfahren „reale und greifbare“ Aussicht auf Entlassung bedeuten könnte. ${ }^{145}$

\section{Die prozessuale Absicherung der Aussicht auf Entlassung}

Mit der materiellen Aussage, dass eine unüberprüfte, unreduzierbare lebenslange Freiheitsstrafe ihrer Natur nach mit Art. 3 EMRK nicht zu vereinbaren ist, ist der wichtigste und lange angemahnte Schritt gegangen. Das Ende des Weges ist damit aber noch nicht erreicht: Die Diskussion wird sich nun auf die Frage einer adäquaten prozessuale Absicherung verlagern. Immerhin ist anhand des Vinter-Falles klar, welches Verfahren und welche Art von Entlassungsaussicht nicht ausreichen - die oben geschilderte derzeitige englische Praxis, die eine Entlassung exklusiv dem Innenminister vorbehält und sich auf Fälle von Krankheiten im Endstadium bzw. schwerster Pflegebedürftigkeit beschränkt, ist mangelhaft. Der Gerichtshof kommentiert spitzzüngig, dass hier schon der Begriff „Entlassung“ fragwürdig ist, wenn es nur darum gehe, den Gefangenen zu Hause sterben zu lassen. ${ }^{146}$

Er sieht sich jedoch wegen des Subsidiaritätsgrundsatzes zu einer genaueren Festschreibung des Verfahrens aus Art. 3 EMRK selbst nicht in der Lage. ${ }^{147}$ In diesem Zusammenhang hat der Gerichtshof die Doktrin vom Beurteilungsspielraum (margin of appreciation) entwickelt, die anhand von inzwischen recht umfangreicher Kasuistik über die Kontrolldichte entscheidet. Nach dem Selbstverständnis des Gerichtshofs liegt eine Überprüfung des Strafrechtssystems, einschließlich der Strafzumessung und Entlassungsvorkehrungen, außerhalb seiner Kompetenz. Dies ist zumindest dann der Fall, wenn „das System nicht gegen die Prinzipien, die in der Konvention aufgestellt sind, verstößt" ${ }^{148}$ - was zirkelschlüssig ist. Den durch die Unvereinbarkeitserklärung der whole life order mit Art. 3 EMRK eingeschlagenen Weg geht die

145 Teilweise abweichendes Votum der Richterinnen und Richter Tulkens, Cabral Barreto, Fura-Sandström, Spielmann und Jebens zum Kafkaris-Urteil (Fn. 121), dort Rn. 6.

146 EGMR (GK), Vinter u.a../. Großbritannien (Fn. 1), Rn. 127.

147 Der anerkannte Subsidiaritätsgrundsatz soll gestärkt werden und wird nicht zuletzt auf britische Initiative explizit in die Präambel der EMRK aufgenommen, wenn das 15. Zusatzprotokoll (CETS 213) vom 24.6.2013 in Kraft gesetzt ist.

148 EGMR (GK), Vinter u.a../. Großbritannien, (Fn. 1), Rn. 104. 
Große Kammer jedenfalls nicht zu Ende, indem sie erklärt, dass es nicht ihre Sache sei, die Verfahrensgarantien festzulegen. ${ }^{149}$

Im Prinzip lassen sich aber so viele Kriterien für die Überprüfung zusammentragen, dass sich ein relativ klares Bild abzeichnet: Das Gewicht des betroffenen Rechtsguts - der Menschenwürde - macht eine transparentes und damit nachprüfbares Verfahren notwendig, der Gerichtshof selbst bringt den Bestimmtheitsgrundsatz ins Spiel. Mit seinem Argument, warum schon bei der Verhängung der nicht reduzierbaren lebenslangen Freiheitsstrafe (und nicht erst im Laufe der Zeit) eine Verletzung von Art. 3 EMRK vorliegt, nennt er gleichzeitig Kriterien für die Überprüfung: Der Gefangene muss wissen, was er tun muss, um für eine Entlassung in Frage zu kommen und unter welchen Bedingungen bzw. wann sie geprüft wird. Damit liegt schon mal fest, dass es Kriterien und Fristen für die Überprüfung geben muss und sie weder vom Gutdünken Dritter noch von „humanitären Erwägungen“ bzw. Voraussetzungen wie lebensbedrohliche Erkrankung oder Siechtum abhängen können. Mit den Ausführungen zur Bedeutung von Resozialisierungsbemühungen ist nicht nur klargestellt, dass der Gefangene hierzu eine Chance haben muss, sondern auch, dass der Überprüfungsmechanismus in der Lage sein muss, sie zu beurteilen. Ebenso ist denkbar, dass eine Gefährlichkeit neu oder sogar erstmals beurteilt werden muss. Hierzu findet sich die Formulierung, dass die Neugewichtung der Strafzwecke erfordert, dass sie „angemessen evaluiert“ 150 werden müssen, was den Überprüfungsmechanismus weiter konturiert: Eine Evaluierung von persönlicher Entwicklung und Gefährlichkeit erfordert Fachkunde. Schließlich äußert die Große Kammer - nachdem sie ausgeführt hat, warum sie zum Verfahren eigentlich nicht Stellung nehmen kann - dass es im europäischen Vergleich und auch im internationalen Strafrecht weitgehende Einigkeit über die Notwendigkeit eines speziellen („dedicated“) Kontrollmechanismus gebe, er in der Regel spätestens nach 25 Jahren greife und danach weitere periodische Überprüfungen beinhalte. Es wäre also durchaus möglich gewesen, Farbe zu bekennen und die Notwendigkeit eines spezifischen Kontrollmechanismus aus Art. 3 EMRK selbst abzuleiten, ähnlich wie es das Bundesverfassungsgericht unter Berufung auf Art. 1 GG gemacht hat.

Fraglich ist weiter, ob ein Gericht über die Weitervollstreckung entscheiden muss. Der deutsche Gesetzgeber hat damals aus den Vorgaben des Bundesverfassungsgerichts diesen Schluss gezogen und das Verfahren zur Entlassung aus lebenslanger Haft weitgehend dem der Entlassung aus zeitiger Freiheitsstrafe angeglichen. Ob dies nach den Rechtsgarantien aus Art. 5 EMRK im Zusammenhang mit der Freiheitsentziehung notwendig ist, ist umstritten. Nach Art. 5 Abs. 4 EMRK kann sich die 
Frage ergeben, ob eine zunächst legitime Freiheitsentziehung im Sinne des Art. 5 Abs. 1 EMRK zu einem späteren Zeitpunkt noch immer rechtmäßig vollstreckt wird.

Dies muss ein Gericht prüfen und, falls es nicht der Fall ist, eine Entlassung anordnen. Bislang hat der Gerichtshof eine Verletzung von Art. 5 Abs. 4 EMRK in den Fällen nicht reduzierbarer lebenslanger Freiheitsstrafen jedoch entweder nicht geprüft oder verneint. ${ }^{151}$ Man wird eine Geltung jedoch annehmen müssen: ${ }^{152}$ Schon bisher ist akzeptiert, dass bei unbestimmten freiheitsentziehenden Strafen und Maßnahmen, z. B. den deutschen Maßregeln nach $\$ 63$ und 66 StGB, von Zeit zu Zeit eine faktische Überprüfung der Voraussetzungen erfolgen muss - und zwar durch ein Gericht. Dies hat der EGMR auch für einen englischen Fall angenommen, in dem nach Verbüßung der Mindestverbüßungszeit eine lebenslange Freiheitsstrafe weiter vollstreckt worden war, weil eine entsprechende Gefährlichkeit des Betroffenen angenommen wurde. ${ }^{153}$ In den Fällen der whole life orders stellt sich dieselbe Frage: Zwar ist es nicht - wie in Fällen mit festgelegtem tariff - zwingend, dass die Weitervollstreckung irgendwann von neuen Strafzwecken wie fortbestehender Gefährlichkeit abhängig gemacht wird, die Strafzwecke der Vergeltung und der Abschreckung gelten möglicherweise fort. Eben dies muss aber geprüft bzw. „angemessen evaluiert“ werden. Fragen der Entwicklung der Persönlichkeit des Gefangenen über einen langen Zeitraum hinweg und etwaige Verschiebungen der Strafzwecke können aber nicht im ursprünglichen Urteil vorweggenommen werden, sind mithin neue Fragen der Rechtmäßigkeit einer Freiheitsentziehung. Über sie darf deshalb kein Exekutivorgan, sondern muss nach Art. 5 Abs. 4 EMRK ein Gericht entscheiden. ${ }^{154}$

Als Frage bleibt, wann eine Überprüfung erfolgen muss. Letztlich bedeutet diese Frage auch, wann die Strafzwecke der Vergeltung und Abschreckung gewissermaßen als verbraucht gelten können. Hier suggeriert der Gerichtshof, dass der Rechtsvergleich eine 25-Jahres-Frist nahe lege. Das ist insofern nicht ganz richtig, als in vielen europäischen Staaten die Überprüfung einer lebenslangen Freiheitsstrafe früher erfolgt. ${ }^{155}$ Auch nach Maßgabe von EU-Recht, in diesem Fall dem Rahmenbeschluss

151 Die Große Kammer konnte im Vinter-Fall ebenso wie im Fall Kafkaris aus formalen Gründen auf Art. 5 Abs. 4 EMRK nicht eingehen. In der Kammerentscheidung Vinter und in einer späteren erneuten Entscheidung im Fall Kafkaris (EGMR, 21.6.2011, 9644/09) war ebenfalls argumentiert worden, dass bei fortgeltendem Strafzweck (punishment), der keinen zeitlichen Veränderungen zugänglich sei, auch die ursprüngliche Verurteilung zu lebenslanger Freiheitsstrafe als Legitimation ausreiche.

152 In Ergebnis ebenso D. van Zyl Smit, P. Weatherby, S. Creighton, Whole Life Sentences and the Tide of European Human Rights Jurisprudence: What Is to Be Done?, Human Rights Law Review, 2014, S. 59 (75 ff.).

153 EGMR vom 28.5.2002 (GK), Stafford./. Großbritannien, Nr. 46295/99, Rn. 87 ff.

154 Entsprechend hatte in einem Minderheitsvotum im Fall Kafkaris Richter Bratza argumentiert, der prinzipiell Art. 5 Abs. 4 EMRK für einschlägig hielt. Nicht völlig klar ist deshalb, weswegen in der Kammerentscheidung im Fall Vinter u.a., an der er beteiligt war, Art. 5 Abs. 4 EMRK relativ zügig als irrelevant abgetan wird (Rn. $102 \mathrm{f}$.).

155 In den 32 aufgeführten Staaten ermöglichen immerhin 20 eine Überprüfung nach spätesten 20 Jahren. 
zum Europäischen Haftbefehl, kann (bzw. muss nach deutschem Recht) die Auslieferung verweigert werden, wenn bei lebenslanger Freiheitsstrafe eine Überprüfung nicht spätestens nach 20 Jahren erfolgt. ${ }^{156}$ Selbst dort, wo in bestimmten Fällen eine spätere Überprüfung vorgesehen ist (neben Deutschland bei besonderer Schwere der Schuld ist dies, soweit ersichtlich, etwa in Irland, Belgien und Frankreich der Fall) erreicht sie nicht immer die 25-Jahres-Frist. Ob man diesen Grenzwert von 25 Jahren aus der Praxis der Internationalen Gerichtshöfe, die qua definitionem nur mit besonders schweren Verbrechen und in der Regel eben auch mit besonders schwerer Schuld zu tun haben, als Vergleichsmaßstab heranziehen kann, ist hingegen fraglich. So würde allerdings in den englischen Fällen an die Praxis vor 2003 angeknüpft werden.

\section{Konsequenzen}

Was bedeutet nun das Urteil für die Praxis der lebenslangen Freiheitsstrafe in Europa? Viele Staaten erfüllen die Kriterien, die der Gerichtshof entwickelt hat bzw. sind in ihrer Ausgestaltung der lebenslangen Freiheitsstrafe deutlich weiter fortgeschritten.

In England jedoch provozierte das Urteil erwartungsgemäß entsetzte Kommentare in Medien und Politik. ${ }^{157}$ Anlass für das Urteil war letztlich aber eine populistische Rechtsänderung der jüngeren Zeit, sehr wahrscheinlich wäre es nach der bis 2003 bestehenden und auch nicht gerade großzügigen Rechtslage gar nicht zu einem Urteil gekommen: Die nach altem Recht vorgesehene Überprüfung nach 25 Jahren wurde gänzlich gestrichen, der Gnadenträger hingegen mit der an Restriktivität nicht zu überbietenden ministeriellen Verordnung (Prison Service Order 4700, siehe oben) zu den Voraussetzungen einer Begnadigung an die Kandare genommen. Einen überzeugenden Grund für diese rechtliche Gestaltung konnte die britische Regierung in Straßburg nicht nennen. Forderungen nach einem Austritt aus dem Kreis der EMRKStaaten mögen als populistische, aber unrealistische Drohungen wahrgenommen werden, bekommen aber durch die offiziellen Reaktionen auf das Urteil neue Nahrung. ${ }^{158}$ Die konkrete Reaktion zur Umsetzung des Urteils könnte nun eine Mini-

156 Art. 5 (2) des Rahmenbeschluss 2002/584/JI des Rats vom 13.6.2002 über den Europäischen Haftbefehl und die Übergabeverfahren zwischen den Mitgliedstaaten, bzw. $\int 83$ Nr. 4 IRG. Ein Gnadenverfahren soll aber ausreichend sein, zumindest wenn es justizförmig ausgestaltet ist (bzgl. einer Auslieferung nach Polen BGH NJW 2012, 2980).

157 In verschiedenen Zeitungen wurde der Premierminister dahingehend zitiert, er sei „very, very, very, very disappointed“ (z. B. Daily Mail, 10.7.2013, S. 7; The Guardian, 10.7.2013, S. 9); Justizminister Grayling äußerte, dass das Urteil die Väter der EMRK in ihren Gräbern rotieren lasse (van Zyl Smit u.a., Fn. 152) und schrieb: "This meddling in our affairs must stop now [Diese Einmischung in unsere Angelegenheiten muss jetzt aufhören]“, Daily Mail, 10.7.2013, S. 7).

158 Sie wurden mehrfach geäußert, sowohl von Premierminister Cameron (z. B. The Guardian „UK may withdraw from European rights convention over Abu Qatada“, Online-Ausgabe vom 24.4.2013) als auch von Innenministerin Theresa May (The Guardian, „Conservatives promise to scrap Human Rights Act after next election“, Online-Ausgabe vom 30.9.2013). 
mallösung sein, die lediglich versucht, die angesprochene Verordnung anzupassen. Alternativ könnte man die Rechtslage von vor 2003 wieder herstellen, die jedoch nach der hier vertretenen Auffassung insofern verändert werden müsste, als ein Gericht zu entscheiden hätte. ${ }^{159}$

Eine Entscheidung des englischen Court of Appeal vom Februar 2014 wurde in der Öffentlichkeit so aufgenommen, als würde in vollem Umfang Recht und Praxis des whole life tariff aufrechterhalten, mithin das Straßburger Urteil nicht umgesetzt. ${ }^{160}$ Dieser Lesart ist entgegengetreten worden: ${ }^{161}$ Das Gericht akzeptiere durchaus das Überprüfungsgebot des EGMR, allerdings sei es der Auffassung, der Secretary of State könne eine konventionskonforme Haftprüfung durchführen. Es mache ihm dabei aber eine Reihe von strengen Auflagen - vor allem soll seine Entscheidung gründlich begründet und überprüfbar sein („must be reasoned by reference to the circumstances of each case and is subject to scrutiny by way of judicial review"). ${ }^{162}$ Wie sich die Praxis dieser in den Händen der Exekutive verbleibenden, aber justiziell immerhin nachprüfbaren Entscheidung entwickeln wird, bleibt abzuwarten. Zu berücksichtigen ist in diesem Zusammenhang zusätzlich, dass englische Gerichte auch bei den anderen lebenslangen Freiheitsstrafen zunehmend längere Mindestverbüßungszeiten festlegen - 30 Jahre und mehr sind keine Seltenheit; in spektakulären Mordfällen der letzten Zeit sind auch 40 und 45 Jahre verhängt worden. ${ }^{163}$

Die geschilderte Rechtslage in den Niederlanden mit ihren restriktiven Kriterien für eine Entlassung gnadenhalber, muss ebenfalls angepasst werden. Der Reformentwurf, der eine justizielle Überprüfung vorsieht, dürfte nunmehr bessere Umsetzungschancen haben. Allerdings zeigt sich hier das Dilemma des impliziten Vorschlags des EGMR: Die Verfasser des Reformentwurfs fordern eine Überprüfung nach 15 Jahren, diesem Vorschlag könnten Gegner entgegenhalten, dass der EGMR offenbar eine Frist von 25 Jahren für ausreichend erachtet.

Für Spanien scheint das Urteil des EGMR zunächst nicht bedeutsam, da es keine lebenslangen Freiheitsstrafen gibt. Wie in Abschnitt II dieses Beitrags dargestellt, ist es nach spanischem Recht bei sehr langen Freiheitsstrafen möglich, dass die Über-

159 Diese beiden Möglichkeiten bringt in einem Minderheitsvotum auch der britische Richter beim EGMR, Mahoney, ins Spiel. Zum Erfordernis einer Entscheidung der Judikative vgl. auch oben Fn. 67 und EGMR vom 20.11.2007, Anderson./.Großbritannien, Nr. 73652/01.

160 BBC News Online vom 18.2.2014: "Court of Appeal upholds principle of whole-life prison terms", www.bbc.com/news/uk-26236225 (abgerufen am 2.5.2014); Regina/Ian McLoughlin und Regina/Lee William Newell, [2014] EWCA Crim 188.

161 D.van Zyl Smit, Whole life imprisonment reconsidered, OUP blog vom 2.3.2014, http://blog.oup.com/ 2014/03/whole-life-imprisonment-reconsidered-pil/ (abgerufen am 4.7.2014).

162 Regina/Ian McLoughlin und Regina/Lee William Newell, [2014] EWCA Crim 188, Rdnr. 34.

163 Vgl. van Zyl Smit u.a. (Fn. 152), S. 59 (65). In den beiden in Fn. 160 genannten verbundenen Fällen waren es 40 Jahre; nach einem gemeinschaftlich auf offener Straße an einem Soldaten verübten Mord waren es 45 Jahre und erneut ein whole life tariff, BBC News online vom 26.2.2014, Lee Rigby murder: Adebolajo and Adebowale jailed, www.bbc.com/news/uk-26357007 (abgerufen am 4.7.2014). 
prüfung der Entlassung unter Umständen erst sehr spät erfolgt - jedenfalls deutlich nach dem Ablauf von 25 Jahren. Ob für die betroffenen Gefangenen diese Option ausreichend Anlass zur Hoffnung im Sinne des Art. 3 EMRK darstellt, bedarf näherer Untersuchung.

Aus deutscher Sicht könnte man auf dem Standpunkt stehen, das europäische Recht setze sich mit einer Frage auseinander, die in Deutschland seit Jahrzehnten keine Rolle mehr spielt; das Urteil sei daher irrelevant oder bestenfalls interessant. Zumindest in Fragen der Auslieferung kann jedoch auch die deutsche Justiz direkt betroffen sein. Die Konsequenzen des EGMR-Urteils, etwa in Bezug auf die Entlassungsaussichten de jure und de facto im Auslieferungsstaat und die Übertragbarkeit dieser Überlegungen auf Nicht-Konventionsstaaten, müssen allerdings im Einzelnen noch diskutiert werden. ${ }^{164}$ Außerdem ist bei der Frage der Aussetzbarkeit von lebenslangen bzw. sehr langen Freiheitsstrafen des Jugoslawien-Gerichthofs die akzeptierte 30-Jahres-Frist, die z. B. das deutsche Gesetz übernommen hat, ebenfalls diskussionswürdig.

\section{E. Ausblick}

Das Vinter-Urteil zeigt einmal mehr, ${ }^{165}$ dass der EGMR die Konvention als lebendiges Instrument ernst nimmt. Art. 3 EMRK wird dabei weiter im Sinne einer allgemeinen Menschenwürdegarantie entwickelt. Die direkte praktische Bedeutung für die Betroffenen mag gering sein, denn das Recht auf eine Überprüfung ihrer lebenslangen Freiheitsstrafen bedeutet noch lange nicht, dass sie ihrer tatsächlichen Freilassung näher kommen, wie auch vom Gerichtshof z. B. in seinen Pressemeldungen immer wieder betont wird. Es ist aber von grundsätzlicher Wichtigkeit, weil Ausweitungstendenzen und dem Einfluss exekutivischer, oftmals populistischer Beweggründe ein Riegel vorgeschoben wird: Wie das englische Beispiel - aber auch das spanische im Bereich der hohen zeitigen Freiheitsstrafen - zeigen, erscheint der Bereich höchster Strafen offenbar stets geeignet, kriminalpolitische Handlungsfähigkeit zu demonstrieren. Dass es deshalb konsequent und geboten gewesen wäre, entsprechend anfälligen Gnadenträgern die Entscheidung über die Entlassung aus der Hand

164 Dass drohende Menschenrechtsverletzungen auf Auslieferungsentscheidungen Auswirkungen haben können, ist seit dem Soering-Urteil des EGMR (NJW 1990, 2183) anerkannt; zur Frage der Auslieferung bei nicht reduzierbaren lebenslangen Freiheitsstrafen vgl. auch BVerfGE 113, $154=$ NJW 2005, 3483 und BVerfG EuGRZ 2010, 256.

165 Der EGMR hat im Urteil v. 20.5.2014, László Magyar/Ungarn, No. 73594/10 inzwischen nochmals in aller Deutlichkeit festgehalten, dass ein zu einer lebenslangen Freiheitsstrafe Verurteilter zu Beginn seiner Freiheitsstrafe wissen muss, unter welchen Bedingungen und wann eine Prüfung seiner Entlassung erfolgen kann bzw. was er dazu tun kann und muss. Wo das nationale Recht eine solche Überprüfung nicht vorsieht, ist ein Verstoß gegen Art. 3 EMRK gegeben (Rn. 53). Darüber hinaus hat der Gerichthof in Ungarn die Gefahr gesehen, dass ein „systemisches Problem “ Anlass zu weiteren entsprechenden Beschwerden geben wird und deshalb Art. 46 EMRK angewendet, wonach die einzige Möglichkeit der adäquaten Abhilfe durch den unterlegenen Staat ist, eine entsprechende Reform in die Wege zu leiten (Rn. 69 ff.). 
zu nehmen, und dies sowohl aus Art. 3 EMRK direkt als auch aus Art. 5 Abs. 4 EMRK abzuleiten, wurde dargelegt.

Das Urteil erbringt nunmehr dennoch den eingangs angesprochenen Beitrag zur europäischen Rechtskultur: Wurde hier bislang als große Errungenschaft vor allem die Abschaffung der Todesstrafe im Geltungsbereich des Europarates genannt, ${ }^{166}$ geht nun das Verbot nicht reduzierbarer lebenslanger Freiheitsstrafen tatsächlich einen Schritt weiter und darf so als zivilisatorischer Fortschritt verstanden werden. Gleichzeitig wird deutlich, dass auf Europaratsebene weitere vereinheitlichende Kräfte wirken, wenn das Gericht zur Untermauerung die Empfehlungen des Europarats oder die Arbeit des Anti-Folter-Komitees heranzieht. Bemerkenswert ist in diesem $\mathrm{Zu}$ sammenhang das Bekenntnis zum Resozialisierungsgedanken unter Rückgriff auf diese Instrumente, aber auch auf nationale Quellen. Aus deutscher Sicht erfreulich ist, dass es in diesem Fall gerade die deutsche Verfassungsrechtsprechung ist, die eine zustimmende Würdigung erfährt, selbst wenn die Verknüpfung von Art. 3 EMRK mit einem Resozialisierungsanspruch allenfalls zurückhaltend erfolgt ist.

Mit der Hinwendung zur Spezialprävention ist jedoch das für die Praxis - und die Betroffenen - überwältigende Problem verbunden: So lange überhaupt periodisch Überprüfungen der Unterbringung stattfinden, wird die die lebenslange Inhaftierung wegen fortbestehender Gefährlichkeit ohne weiteres als konventionsgemäß akzeptiert. Der Risikoprognose kommt damit ein immer größeres Gewicht zu. Da auch sie kriminalpolitisch keineswegs immun ist, dürften sich der kriminalpolitische Druck auf sie bzw. diejenigen, die sie erstellen und über sie urteilen müssen, noch weiter verstärken. „Wegsperren für immer“ hat Konjunktur in Europa und mag auch das deutsche Beispiel in Bezug auf lebenslange Freiheitsstrafe leuchtend erscheinen, die jüngere Praxis der Sicherungsverwahrung oder andere Unterbringungsformen gefährlicher Täter ist es nicht. ${ }^{167}$

Das Vinter-Urteil und sein Hintergrund offenbaren außerdem nach wie vor erhebliche Unterschiede zwischen den europäischen Staaten bei der Gewichtung von Strafzwecken und dem, was bei Strafen als angemessen empfunden wird. Das zeigen die sehr hohen zeitigen Freiheitsstrafen, die in manchen Staaten möglich sind. An die Frage nach der humanen Ausgestaltung lebenslanger Freiheitsstrafe wird sich deshalb diejenige anschließen müssen, die auf sehr lange Freiheitsstrafen bzw. sehr lange Mindestverbüßungszeiten zielt.

166 Vgl. Nachweise in Fn. 1; D. Garland, Peculiar Institution, America's death penalty in an age of abolition, Cambridge: 2010, S. $101 \mathrm{ff}$.

167 Hierzu ausführlich Drenkhahn/Morgenstern, Dabei soll es uns auf den Namen nicht ankommen Der Streit um die Sicherungsverwahrung. ZStW (124) 2012, S. 123-203. 